\title{
AN EFFICIENT HIGH-ORDER NYSTRÖM SCHEME FOR ACOUSTIC SCATTERING BY INHOMOGENEOUS PENETRABLE MEDIA WITH DISCONTINUOUS MATERIAL INTERFACE
}

\author{
AKASH ANAND, AMBUJ PANDEY, B. V. RATHISH KUMAR, JAGABANDHU PAUL
}

\begin{abstract}
This text proposes a fast, rapidly convergent Nyström method for the solution of the Lippmann-Schwinger integral equation that mathematically models the scattering of time-harmonic acoustic waves by inhomogeneous obstacles, while allowing the material properties to jump across the interface. The method works with overlapping coordinate charts as a description of the given scatterer. In particular, it employs "partitions of unity" to simplify the implementation of highorder quadratures along with suitable changes of parametric variables to analytically resolve the singularities present in the integral operator to achieve desired accuracies in approximations. To deal with the discontinuous material interface in a high-order manner, a specialized quadrature is used in the boundary region. The approach further utilizes an FFT based strategy that uses equivalent source approximations to accelerate the evaluation of large number of interactions that arise in the approximation of the volumetric integral operator and thus achieves a reduced computational complexity of $O(N \log N)$ for an $N$-point discretization. A detailed discussion on the solution methodology along with a variety of numerical experiments to exemplify its performance in terms of both speed and accuracy are presented in this paper.
\end{abstract}

\section{INTRODUCTION}

The solution of direct acoustic scattering problem where the goal is to obtain the scattered wave produced as a result of an interaction between a given incident wave and a given bounded inhomogeneity continues to constitute one of the most challenging problems in computational science, especially where applications of interest require such computations to be carried out for large penetrable scatterers with complex geometries. Formally, the $d$-dimensional direct acoustic scattering problem that we consider in this paper for $d=2$ and 3 is described as follows: given an obstacle $\Omega$, a bounded open subset of $\mathbb{R}^{d}$, with a smooth boundary $\partial \Omega$, and an incident time-harmonic acoustic wave $\mathfrak{u}^{i}$ satisfying

$$
\Delta \mathfrak{u}^{i}(\boldsymbol{x})+\kappa^{2} \mathfrak{u}^{i}(\boldsymbol{x})=0, \quad \boldsymbol{x} \in \mathbb{R}^{d},
$$

where $\kappa=\omega / c_{0}$ is the wavenumber, $\omega$ is the angular frequency, and $c_{0}$ is the constant speed of wave outside the inhomogeneity $\Omega$, find the total acoustic field $\mathfrak{u}$ that satisfies [1]

$$
\Delta \mathfrak{u}(\boldsymbol{x})+\kappa^{2} n^{2}(\boldsymbol{x}) \mathfrak{u}(\boldsymbol{x})=0, \quad \boldsymbol{x} \in \mathbb{R}^{d},
$$

with the refractive index $n(\boldsymbol{x})=c_{0} / c(\boldsymbol{x})$, where $c$, the speed of acoustic wave, is allowed to vary with position within $\Omega$ and the scattered field $\mathfrak{u}^{s}:=\mathfrak{u}-\mathfrak{u}^{i}$ satisfies Sommerfeld radiation condition

$$
\lim _{r \rightarrow \infty} r^{(d-1) / 2}\left(\frac{\partial \mathfrak{u}^{s}}{\partial r}-i k \mathfrak{u}^{s}\right)=0
$$

where $r=\|\boldsymbol{x}\|_{2}=\sqrt{\sum_{j=1}^{d} x_{j}^{2}}$. 
One of the main challenges in obtaining numerical approximation of the solution to the scattering problem arise from the need to accurately describe highly oscillatory functions in large domains. As a certain fixed number of points is necessary to resolve a wavelength, we typically require a large computational grid to obtain any meaningful solution and any effort to further reduce the computational errors demands that the size of discretization grows proportionally in all dimensions. It is, therefore, highly desirable to devise numerical methodologies that are efficient as well as high-order accurate. In this direction, several methodologies, based on diverse formulations of the problem, ranging from differential equation to variational form and to integral equation, have been proposed by various authors. In the solution strategies that are based on differential equation or their corresponding weak formulations $[2,3,4,45,6,7,8,9,10$, , a relatively large computational domain containing the scatterer must be used, together with appropriate absorbing boundary conditions on the boundary of the computational domain. Thus, these procedures often require a large number of unknowns and, hence, lead to large linear systems. In addition, accurate absorbing boundary conditions with efficient numerical implementations are quite difficult to construct; the error associated with such boundary conditions typically dominates the error in the computed solution.

In contrast, the integral equation approach, where the mathematical formulation directly ensures that the solution satisfies condition (3) by suitably employing the radiating fundamental solution, is free from considerations mentioned above, and consequently, does not require solution strategies to discretize outside the inhomogeneity. We, therefore, base our numerical treatment of the scattering problem on an equivalent integral equation formulation which is given by the Lippmann-Schwinger equation [1, 11],

$$
\mathfrak{u}(\boldsymbol{x})+\kappa^{2} \int_{\mathbb{R}^{d}} G_{\kappa}(\boldsymbol{x}, \boldsymbol{y}) m(\boldsymbol{y}) \mathfrak{u}(\boldsymbol{y}) d \boldsymbol{y}=\mathfrak{u}^{i}(\boldsymbol{x}), \quad \boldsymbol{x} \in \mathbb{R}^{d}
$$

where

$$
G_{\kappa}(\boldsymbol{x}, \boldsymbol{y})= \begin{cases}\frac{i}{4} H_{0}^{1}(\kappa|\boldsymbol{x}-\boldsymbol{y}|), & \text { in } \mathbb{R}^{2}, \\ \exp (i \kappa|\boldsymbol{x}-\boldsymbol{y}|) / 4 \pi|\boldsymbol{x}-\boldsymbol{y}|, & \text { in } \mathbb{R}^{3},\end{cases}
$$

is the radiating fundamental solution of Helmholtz equation in the free space and $m(\boldsymbol{x})=1-n^{2}(\boldsymbol{x})$.

In recent years, a lot of progress has been made toward numerical solution of the LippmannSchwinger equation; for example, see [12, 13, 14, 15, 16, 17, 18, 19, 20, 21, 22, 23, 24, 25, 26, 27]. Most fast numerical schemes among these, though high order accurate for smooth scattering media, exhibit only linear convergence in the presence of material discontinuity [12, 15, 17, 22, 26, 27, For example, in [26], Duan and Rokhlin, introduced an efficient high order quadrature formula which utilizes a corrected Trapezoidal rule in conjunction with FFT for fast and accurate approximation of volume integration in (4). The high order convergence of the scheme, however, requires the refractive index $n(\boldsymbol{x})$ to be globally smooth. More recently, a high order direct solver with $O\left(N^{3 / 2}\right)$ computational complexity has been proposed in [27. Again, while this algorithm is robust and provide accurate approximation for smooth scatterer, it does not exhibit rapid convergence in the presence of a discontinuous material interface. A couple of fast techniques, one that rely on the use of "discontinuous FFT" 24] for efficient computations while the other uses FFT for accelerated evaluation of convolution in polar coordinates after a suitable decomposition of the Green's function via addition theorem [19, however, achieve second order accuracy for discontinuous scattering configurations. Another approach for solution of acoustic volumetric scattering problem introduced 
in [13, 14], though high-order convergent, is designed to be computationally efficient only for "thin" scattering configurations. This scheme gains high-order convergence through a combination of changes of parametric variables (in order to resolve the singularities present in the Green function) and by suitably employing "partitions of unity" to yield smooth and periodic integrand away from vicinity of target points. Our present approach, in fact, is a non trivial extension of ideas presented in [13, 14] wherein we obtain a solver for general scattering configurations that exhibits computational complexity of $O(N \log N)$ with respect to the grid size $N$ while retaining high-order accuracy even in the presence of material discontinuity. We believe that this algorithm is the first fast integral equation solver which provide high-order convergence for full volumetric scattering problem with a discontinuous material interface.

The rest of the paper is organized as follows. In Section 2, we present main algorithmic components of our numerical scheme. We then present, in Section 3, a detailed account of our approximation strategy for the integral operator arising in the context of two dimensional volumetric scattering problem. The last part of this section presents a series of numerical experiments to exemplify the performance of the proposed method, both in terms of accuracy and in terms of computational efficiency. This is followed, in Section 4, by a brief discussion on numerical solution of corresponding three dimensional scattering problems using a straightforward extension of our two dimensional solver and demonstrate its effectiveness via some numerical experiments. Finally, our conclusions are summarized in Section 5 .

\section{Principal Components of the method}

As mentioned earlier, we base our numerical strategy on solving the linear system arising out of a Nyström discretization of the Lippmann-Schwinger integral equation. Given the denseness of resulting linear systems where use of direct linear solvers can be prohibitively expensive, we employ the matrix-free version of the iterative solver GMRES [28], in fully complex arithmetic, for the solution of the discrete form of (4). In this context, we focus our presentation on the computational technique for accurate and efficient evaluation of the integral operator

$$
\mathcal{K}[\mathfrak{u}](\boldsymbol{x})=\int_{\Omega} G_{\kappa}(\boldsymbol{x}, \boldsymbol{y}) m(\boldsymbol{y}) \mathfrak{u}(\boldsymbol{y}) d \boldsymbol{y} .
$$

We start by describing the scatterer $\Omega$ through a collection $\mathcal{P}=\left\{\mathcal{P}_{k}\right\}_{k=1}^{K}$ of $K$ overlapping coordinate patches where the $k$-th patch is homeomorphic to an open set $\mathcal{H}_{k} \subset(0,1)^{d}$ via a smooth invertible parametrization $\boldsymbol{\xi}_{k}$. This, in conjunction with a partitions of unity (POU) subordinated to the covering $\mathcal{P}$, that is, functions $\omega_{k}(\boldsymbol{x}): k=1, \ldots, K$, satisfying

$$
\sum_{k=1}^{K} \omega_{k}(\boldsymbol{x})=1 \text { for all } \boldsymbol{x} \in \Omega
$$

where for each $k, \omega_{k} \in C^{\infty}(\Omega)$ with its support contained in $\mathcal{P}_{k}$, reduces the evaluation of (6) to computation of integrals over these $K$ patches. Note here that for all those patches $\mathcal{P}_{k}$ whose closure does not intersect with the boundary of $\Omega$, the corresponding $\omega_{k}$ vanishes to high order along with all of its derivatives on the boundary of the patch. On remaining patches, however, where one of the edges coincide with the boundary of $\Omega, \omega_{k}$ clearly does not vanish and, in fact, attains the value 1 (see Figure 1 for examples). For the clarity of presentation of the proposed quadrature, and to distinguish between patches based on this criterion, we introduce two index 

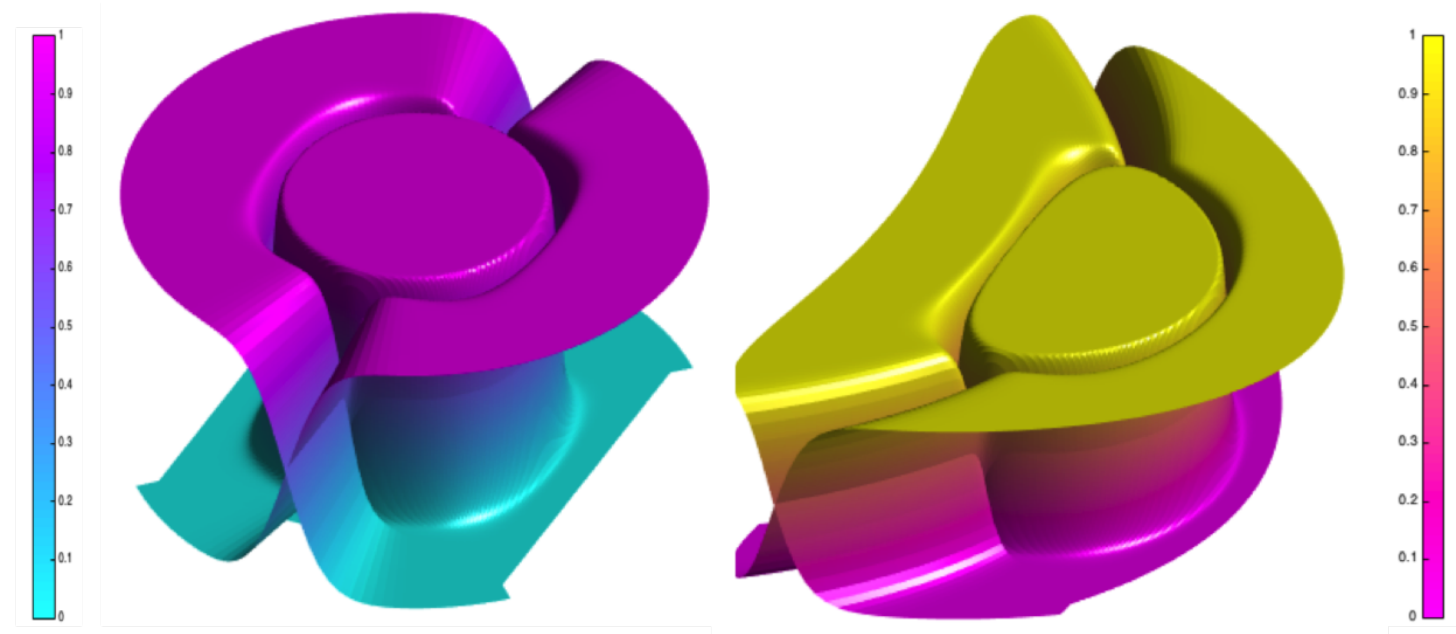

Figure 1. Partitions of unity.

sets, namely, $\mathcal{I}_{I}=\left\{k \mid \overline{\mathcal{P}_{k}} \cap \partial \Omega=\varnothing\right\}$ and $\mathcal{I}_{B}=\left\{k \mid \overline{\mathcal{P}_{k}} \cap \partial \Omega \neq \varnothing\right\}$ corresponding to interior and boundary patches respectively. Now, clearly, the integral (6) can be reexpressed as a sum of integrals over boundary and interior patches

$$
\mathcal{K}[\mathfrak{u}](\boldsymbol{x})=\sum_{k \in \mathcal{I}_{B}} \int_{\mathcal{P}_{k}} G_{\kappa}(\boldsymbol{x}, \boldsymbol{y}) m(\boldsymbol{y}) \mathfrak{u}(\boldsymbol{y}) \omega_{k}(\boldsymbol{y}) d \boldsymbol{y}+\sum_{k \in \mathcal{I}_{I}} \int_{\mathcal{P}_{k}} G_{\kappa}(\boldsymbol{x}, \boldsymbol{y}) m(\boldsymbol{y}) \mathfrak{u}(\boldsymbol{y}) \omega_{k}(\boldsymbol{y}) d \boldsymbol{y} .
$$

In view of this, we introduce a set of quadrature points on the $k$-th patch, say $\mathfrak{T}_{k}$, by taking the image of the equi-spaced grid $\left\{\left(i_{1} / N_{1}, \ldots, i_{d-1} / N_{d-1}, i_{d} / N_{d}\right) \mid 0 \leq i_{\ell} \leq N_{\ell}, \ell=1, \ldots, d\right\}$ in $[0,1]^{d}$ under the map $\boldsymbol{\xi}_{\mathrm{k}}$. Further, the union of these grid points, that is,

$$
\mathfrak{T}=\bigcup_{k=1}^{K} \mathfrak{T}_{k}
$$

where

$$
\mathfrak{T}_{k}=\left\{\boldsymbol{\xi}_{k}\left(i_{1} / N_{1}, \ldots, i_{d-1} / N_{d-1}, i_{d} / N_{d}\right) \mid 0 \leq i_{\ell} \leq N_{\ell}, \ell=1, \ldots, d\right\}
$$

defines the complete set of Nyström discretization points where we seek to approximate the solution of equation (4).

Indeed, the performance of our iterative solver hinges on our ability to approximate the integrals in (7) accurately, in a computationally efficient manner. In the next section, we provide a detailed description of a fast, high order integration scheme in two dimensions that can be readily extended to three dimensions, which we briefly outline in Section 4.

Remark 1. In this text, we often refer to $\boldsymbol{x}$ in equation (7) as a target point whereas points $\boldsymbol{y}$ therein have sometimes been called source points.

Remark 2. While discussing the approximations on boundary patches, we identify the d-th coordinate variable in $[0,1]^{d}$ with the transverse parameter. We, therefore, use the notation $\boldsymbol{t}=$ 
$\left(t_{1}, \ldots, t_{d-1}, t_{d}\right)$ to denote a point in the corresponding parameter space and assume that the boundary coincides with $t_{d}=0$.

\section{FASt AND ACCURATE EVAluation of integrals in TWO Dimensions}

The difficulty in accurately computing integrals in (7) is significantly more when the target point $\boldsymbol{x}$ lies in the integration patch $\mathcal{P}_{k}$ compared to the case when it does not. Indeed, when $\boldsymbol{x} \in \mathcal{P}_{k}$, owing to the singularity of the kernel $G_{\kappa}(\boldsymbol{x}, \boldsymbol{y})$ at $\boldsymbol{y}=\boldsymbol{x}$, the integrand is unbounded within the integration domain and direct use of a standard quadratures yield inaccurate approximations. Thus, specialized quadrature rules must be developed and used to deal with such singular integrals. The case when $\boldsymbol{x} \notin \mathcal{P}_{k}$, in contrast, does not present this challenge. To effectively present these two contrasting scenarios, we further refine the index sets $\mathcal{I}_{B}$ and $\mathcal{I}_{I}$ by introducing target point dependent index sets $\mathcal{M}_{B}(\boldsymbol{x})=\left\{k \in \mathcal{I}_{B} \mid \boldsymbol{x} \in \mathcal{P}_{k}\right\}$ and $\mathcal{M}_{I}(\boldsymbol{x})=\left\{k \in \mathcal{I}_{I} \mid \boldsymbol{x} \in \mathcal{P}_{k}\right\}$ to rewrite (7) as

$$
\begin{aligned}
\mathcal{K}[\mathfrak{u}](\boldsymbol{x}) & =\left(\sum_{k \in \mathcal{M}_{B}(\boldsymbol{x})}+\sum_{k \notin \mathcal{M}_{B}(\boldsymbol{x})}\right) \int_{\mathcal{P}_{k}} G_{\kappa}(\boldsymbol{x}, \boldsymbol{y}) m(\boldsymbol{y}) \mathfrak{u}(\boldsymbol{y}) \omega_{k}(\boldsymbol{y}) d \boldsymbol{y} \\
& +\left(\sum_{k \in \mathcal{M}_{I}(\boldsymbol{x})}+\sum_{k \notin \mathcal{M}_{I}(\boldsymbol{x})}\right) \int_{\mathcal{P}_{k}} G_{\kappa}(\boldsymbol{x}, \boldsymbol{y}) m(\boldsymbol{y}) \mathfrak{u}(\boldsymbol{y}) \omega_{k}(\boldsymbol{y}) d \boldsymbol{y} .
\end{aligned}
$$

Clearly, each of the integrals above can be rewritten in the parametric coordinates

$$
\int_{\mathcal{P}_{k}} G_{\kappa}(\boldsymbol{x}, \boldsymbol{y}) m(\boldsymbol{y}) \mathfrak{u}(\boldsymbol{y}) \omega_{k}(\boldsymbol{y}) d \boldsymbol{y}=\iint_{[0,1]^{2}} G_{\kappa}\left(\boldsymbol{x}, \boldsymbol{\xi}_{k}\left(t_{1}, t_{2}\right)\right) \varphi_{k}[\mathfrak{u}]\left(t_{1}, t_{2}\right) \xi_{k}^{\prime}\left(t_{1}, t_{2}\right) d t_{1} d t_{2},
$$

where $\varphi_{k}[\mathfrak{u}]\left(t_{1}, t_{2}\right)=m\left(\boldsymbol{\xi}_{k}\left(t_{1}, t_{2}\right)\right) \mathfrak{u}\left(\boldsymbol{\xi}_{k}\left(t_{1}, t_{2}\right)\right) \omega_{k}\left(\boldsymbol{\xi}_{k}\left(t_{1}, t_{2}\right)\right)$, and $\xi_{k}^{\prime}$ denotes the Jacobian of the transformation $\boldsymbol{\xi}_{k}$.

Now, for the cases $k \notin \mathcal{M}_{B}(\boldsymbol{x})$ and $k \notin \mathcal{M}_{I}(\boldsymbol{x})$ in $(10), G_{\kappa}(\boldsymbol{x}, \boldsymbol{y})$ remains non-singular throughout the region of integration. Though adopting a single high-order approximation scheme for both scenarios is possible, we actually utilize two different quadratures to take advantage of a more favorable behavior of the integrands in the later case when $k \notin \mathcal{M}_{I}(\boldsymbol{x})$ where $\varphi_{k}[\mathfrak{u}]$ vanish to high order at the boundary of the integration domain and have smooth periodic extensions to $\mathbb{R}^{2}$. As is well known, the trapezoidal rule exhibits super-algebraic convergence for smooth and periodic integrands, which we indeed employ to obtain accurate approximations in this case. In contrast, when $k \notin \mathcal{M}_{B}(\boldsymbol{x})$, a straightforward use of trapezoidal rule does not produce highorder accuracy as the integrands do not vanish at $t_{2}=0$. We overcome this minor difficulty by utilizing the trapezoidal rule for the integration with respect to $t_{1}$-variable while employing a composite Newton-Cotes quadrature in transverse variable $t_{2}$ to achieve approximations whose rate of convergence directly depends on the order of Newton-Cotes used and could be enhanced arbitrarily as long as the smoothness of $m$ within $\Omega$ allows it.

As mentioned above, integrands in (10) corresponding to the cases when $k \in \mathcal{M}_{B}(\boldsymbol{x})$ and $k \in \mathcal{M}_{I}(\boldsymbol{x})$ are singular. To achieve rapidly convergent approximations, we rely on analytic resolution of singularities through suitable changes of parametric variables and application of high-order quadratures to resulting smooth integrands. In addition, we also localize the region where such coordinate transformations are affected to a small neighborhood of the singular point using a suitable 
smooth and compactly supported cut-off function. Indeed, we see that

(11)

$\iint_{[0,1]^{2}} G_{\kappa}\left(\boldsymbol{x}, \boldsymbol{\xi}_{k}(\boldsymbol{t})\right) \cdots d \boldsymbol{t}=\iint_{[0,1]^{2}} G_{\kappa}\left(\boldsymbol{x}, \boldsymbol{\xi}_{k}(\boldsymbol{t})\right) \cdots \eta\left(\boldsymbol{t} ; \boldsymbol{\xi}_{k}^{-1}(\boldsymbol{x})\right) d \boldsymbol{t}+\iint_{[0,1]^{2}} G_{\kappa}\left(\boldsymbol{x}, \boldsymbol{\xi}_{k}(\boldsymbol{t})\right) \cdots\left(1-\eta\left(\boldsymbol{t} ; \boldsymbol{\xi}_{k}^{-1}(\boldsymbol{x})\right)\right) d \boldsymbol{t}$,

where $\eta\left(\cdot ; \boldsymbol{t}_{0}\right)$ is a $C^{\infty}$ function that it is compactly supported in a neighborhood of the point $\boldsymbol{t}_{0} \in[0,1]^{2}$ while $\eta \equiv 1$ in a smaller neighborhood of $\boldsymbol{t}_{0}$. This localization of singularity as seen in the first integral on the right hand side of (11) brings in a two-fold benefit, namely, (a) it limits the relatively expensive treatment of singularity which, among other computational challenges, also demands an interpolation of grid-data to off-grid quadrature points to a small integration domain, and (b) it allows for additional speed-up in the computation of non-singular second term on the right hand side of (11). Note that this regular integral, of course, can be integrated to high-order using the same numerical quadratures that we apply in the case of $\boldsymbol{x} \notin \mathcal{P}_{k}$, as explained above.

It is straightforward to see that the direct application of the integration scheme that we described above leads to a computational complexity of $O\left(N^{2}\right)$, where $N=|\mathfrak{T}|$ is the size of the set $\mathfrak{T}$, the total number of quadrature points in the Nyström scheme. However, as we outline below, one can improve the computational complexity of this methodology to $O(N \log N)$ by breaking the overall computation of these integrals into two parts - a relatively small but specialized calculation in the neighborhood of singularity and remaining non-singular contributions arising from the voluminous bulk. Toward this, we begin by bounding the inhomogeneity $\Omega$ by a square cell $\mathcal{C}$ of side length $A$. This cell is further partitioned into $L^{2}$ identical cells $\mathcal{C}_{i j}(i, j=1, \cdots, L)$ of side length $H=A / L$ such that the bounding cell $\mathcal{C}$ contains $L$ of these smaller cells along its sides (for an example, see Figure 2). We assume, without loss of generality, that for the chosen side length $A$, cells $\mathcal{C}_{i j}$ do not admit inner acoustical resonance. This, of course, can be easily ensured by necessary adjustment in the choice of $A$ so that $-\kappa^{2}$ is not a Dirichlet eigenvalue of the Laplace operator in the cell $\mathcal{C}_{i j}$. These cells are used to break the computation of integrals appearing in equation (10) into adjacent and non-adjacent calculations which, as pointed out above, is primarily motivated by our desire for the method to have a more favorable computational complexity than the quadratic cost in the number of unknowns.

We say a source point $\boldsymbol{y}$ is adjacent to a fixed point target point $\boldsymbol{x} \in \mathcal{C}_{i j}$, if it belongs to the set $\mathcal{N}(\boldsymbol{x})$ defined by

$$
\mathcal{N}(\boldsymbol{x})=\left\{\boldsymbol{y} \mid \boldsymbol{y} \in C_{k l} \text { for some } k, l \text { satisfying }|k-i| \leq 1,|l-j| \leq 1\right\} .
$$

Obviously, we say $\boldsymbol{y}$ is non-adjacent to $\boldsymbol{x}$ if $\boldsymbol{y} \notin \mathcal{N}(\boldsymbol{x})$. Based on this, we separate the integrals over $\mathcal{P}_{k}$ in 10$)$ into integrals over $\mathcal{P}_{k} \cap \mathcal{N}(\boldsymbol{x})$ accounting for the adjacent contributions and the computationally large non-adjacent contributions for which a variant of the two face FFT based acceleration strategy introduced in [29] is employed for efficient calculations.

We, thus, split integrals in 10 to rewrite the expression for $\mathcal{K}[\mathfrak{u}](\boldsymbol{x})$ as

$$
\begin{aligned}
\mathcal{K}[\mathfrak{u}](\boldsymbol{x}) & =\left\{\left(\sum_{k \in \mathcal{M}_{B}(\boldsymbol{x})}+\sum_{k \notin \mathcal{M}_{B}(\boldsymbol{x})}\right) \int_{\mathcal{P}_{k} \cap \mathcal{N}(\boldsymbol{x})}+\sum_{k \in \mathcal{I}_{B}} \int_{\mathcal{P}_{k} \cap \mathcal{N}(\boldsymbol{x})^{c}}\right\} G_{\kappa}(\boldsymbol{x}, \boldsymbol{y}) m(\boldsymbol{y}) \mathfrak{u}(\boldsymbol{y}) \omega_{k}(\boldsymbol{y}) d \boldsymbol{y} \\
+ & \left\{\left(\sum_{k \in \mathcal{M}_{I}(\boldsymbol{x})}+\sum_{k \notin \mathcal{M}_{I}(\boldsymbol{x})}\right) \int_{\mathcal{P}_{k} \cap \mathcal{N}(\boldsymbol{x})}+\sum_{k \in \mathcal{I}_{I}} \int_{\mathcal{P}_{k} \cap \mathcal{N}(\boldsymbol{x})^{c}}\right\} G_{\kappa}(\boldsymbol{x}, \boldsymbol{y}) m(\boldsymbol{y}) \mathfrak{u}(\boldsymbol{y}) \omega_{k}(\boldsymbol{y}) d \boldsymbol{y},
\end{aligned}
$$


where $\mathcal{N}(\boldsymbol{x})^{c}$ denotes the compliment of $\mathcal{N}(\boldsymbol{x})$, that is, the set $\mathfrak{T} \backslash \mathcal{N}(\boldsymbol{x})$.

We note here that the computational cost of carrying out the adjacent calculations for all target points $\boldsymbol{x} \in \mathfrak{T}$, in view of the fact that on an average each cell contain $O\left(N / L^{2}\right)$ discretization points, is $N \times O\left(N / L^{2}\right)=O\left(N^{2} / L^{2}\right)$.

The cost involved in computing the contributions to integrals coming from $\mathcal{P}_{k} \cap \mathcal{N}(\boldsymbol{x})^{c}$ for all target points $\boldsymbol{x} \in \mathfrak{T}$, as we explain later in Section $\sqrt{3.2}$ is given by $O\left(L N^{1 / 2} \log \left(L N^{1 / 2}\right)\right)+$ $O\left(N^{3 / 2} / L^{3}\right)+O\left(N^{3 / 2} / L\right)$. Thus, we get

$$
O\left(L N^{\frac{1}{2}} \log \left(L N^{\frac{1}{2}}\right)\right)+O\left(\frac{N^{\frac{3}{2}}}{L^{3}}\right)+O\left(\frac{N^{\frac{3}{2}}}{L}\right)+O\left(\frac{N^{2}}{L^{2}}\right)
$$

as the total cost of computing $\mathcal{K}[\mathfrak{u}]$ at all Nyström nodes. This, of course, suggests that by choosing the parameter $L=O\left(N^{1 / 2}\right)$, the computational complexity of the algorithm reduces to the desired $O(N \log N)$.

3.1. Singular Integration. Recall that when the target point $\boldsymbol{x}$ belongs to the integration patch $\mathcal{P}_{k}$, we break the integral as follows:

$$
\begin{aligned}
& \int_{\mathcal{P}_{k}} G_{\kappa}(\boldsymbol{x}, \boldsymbol{y}) m(\boldsymbol{y}) \mathfrak{u}(\boldsymbol{y}) \omega_{k} d \boldsymbol{y}=\iint_{[0,1]^{2}} G_{\kappa}\left(\boldsymbol{x}, \boldsymbol{\xi}_{k}(\boldsymbol{t})\right) \varphi_{k}[\mathfrak{u}](\boldsymbol{t}) \xi_{k}^{\prime}(\boldsymbol{t})(\boldsymbol{y}) \eta\left(\boldsymbol{t} ; \boldsymbol{\xi}_{k}^{-1}(\boldsymbol{x})\right) d \boldsymbol{t}+ \\
& \quad \iint_{\boldsymbol{\xi}_{k}^{-1}\left(\mathcal{P}_{k} \cap \mathcal{N}(\boldsymbol{x})\right)} G_{\kappa}\left(\boldsymbol{x}, \boldsymbol{\xi}_{k}(\boldsymbol{t})\right) \varphi_{k}[\mathfrak{u}](\boldsymbol{t}) \xi_{k}^{\prime}(\boldsymbol{t})(\boldsymbol{y})\left(1-\eta\left(\boldsymbol{t} ; \boldsymbol{\xi}_{k}^{-1}(\boldsymbol{x})\right)\right) d \boldsymbol{t}+ \\
& \quad \int_{\mathcal{P}_{k} \cap \mathcal{N}(\boldsymbol{x})^{c}} G_{\kappa}(\boldsymbol{x}, \boldsymbol{y}) m(\boldsymbol{y}) \mathfrak{u}(\boldsymbol{y}) \omega_{k}(\boldsymbol{y})\left(1-\eta\left(\boldsymbol{\xi}_{k}^{-1}(\boldsymbol{y}) ; \boldsymbol{\xi}_{k}^{-1}(\boldsymbol{x})\right)\right) d \boldsymbol{y} .
\end{aligned}
$$

As mentioned above, we rely on the FFT-accelerator for computation of the third term in (14). This, however, entails that we compute this integral as a convolution which, in turn, requires that the integrand remains independent of localization cut-off function $\eta$. This, of course, can easily be achieved by ensuring that the support of $\eta\left(\cdot, \xi_{k}^{-1}(\boldsymbol{x})\right)$ is contained in $\mathcal{N}(\boldsymbol{x})$. The second term in this expression, which we refer to as local correction to the accelerated numerics, is computationally small. We delay a more detailed discussion on these aspects to Section (3.2). We detail the approximation of the singular integration

$$
\mathcal{K}_{k}^{\text {sing }}[\mathfrak{u}](\boldsymbol{x})=\iint_{[0,1]^{2}} G_{\cap \operatorname{supp} \eta}\left(\boldsymbol{x}, \boldsymbol{\xi}_{k}(\boldsymbol{t})\right) \varphi_{k}[\mathfrak{u}](\boldsymbol{t}) \xi_{k}^{\prime}(\boldsymbol{t})(\boldsymbol{y}) \eta\left(\boldsymbol{t} ; \boldsymbol{\xi}_{k}^{-1}(\boldsymbol{x})\right) d \boldsymbol{t},
$$

the first term on the right hand side of equation (14), next.

3.1.1. Singular integration over interior patches. In this section, we describe high-order quadrature rule for evaluation of $\mathcal{K}_{k}^{\text {sing }}[\mathfrak{u}](\boldsymbol{x})$ when $k \in \mathcal{M}_{I}(\boldsymbol{x})$. In this case, following [29], we use the localization function $\eta\left(\boldsymbol{t} ; \boldsymbol{\xi}_{k}^{-1}(\boldsymbol{x})\right)=\chi\left(\left|\boldsymbol{t}-\boldsymbol{\xi}_{k}^{-1}(\boldsymbol{x})\right| / r\right)$ with an appropriate real number $r>0$ and a smooth function $\chi$ such that $\chi \equiv 1$ in a neighborhood of 0 and $\chi(s)=0$ for all $s \geq 1$. We extend the domain of integration in 15$)$ to the disc of radius $r$ around $\boldsymbol{\xi}_{k}^{-1}(\boldsymbol{x})=\left(t_{1}^{\boldsymbol{x}}, t_{2}^{\boldsymbol{x}}\right)$ for each target point $\boldsymbol{x} \in \mathcal{P}_{k} \cap \mathfrak{T}$. Obviously, because of the fact that $\varphi_{k}[\mathfrak{u}]$ vanishes to high order in all directions 
on the boundary of the integration domain, this process does not compromise on the smoothness of the integrand. To perform the integration, we then change to polar coordinates centered at $\left(t_{1}^{x}, t_{2}^{x}\right)$ :

$$
t_{1}=t_{1}^{x}+\rho \cos \theta, t_{2}=t_{2}^{x}+\rho \sin \theta .
$$

If we let $\tilde{\varphi}_{k}[\mathfrak{u}]\left(t_{1}^{\boldsymbol{x}}+\rho \cos \theta, t_{2}^{\boldsymbol{x}}+\rho \sin \theta\right)=$

$$
\varphi_{\mathrm{k}}[\mathfrak{u}]\left(t_{1}^{\boldsymbol{x}}+\rho \cos \theta, t_{2}^{\boldsymbol{x}}+\rho \sin \theta\right) \xi_{k}^{\prime}\left(t_{1}^{\boldsymbol{x}}+\rho \cos \theta, t_{2}^{\boldsymbol{x}}+\rho \sin \theta\right) \eta\left(\left(t_{1}^{\boldsymbol{x}}+\rho \cos \theta, t_{2}^{\boldsymbol{x}}+\rho \sin \theta\right) ;\left(t_{1}^{\boldsymbol{x}}, t_{2}^{\boldsymbol{x}}\right)\right),
$$

then the integral (15) takes the form

$$
\mathcal{K}_{\mathrm{k}}^{\text {sing }}[\mathfrak{u}](\boldsymbol{x})=\frac{1}{2} \int_{0}^{2 \pi} d \theta \int_{-r_{1}}^{r_{1}}|\rho| G_{\kappa}\left(\boldsymbol{x}, \boldsymbol{\xi}_{k}\left(t_{1}^{\boldsymbol{x}}+\rho \cos \theta, t_{2}^{\boldsymbol{x}}+\rho \sin \theta\right)\right) \tilde{\varphi}_{k}[\mathfrak{u}]\left(t_{1}^{\boldsymbol{x}}+\rho \cos \theta, t_{2}^{\boldsymbol{x}}+\rho \sin \theta\right) d \rho .
$$

The appearance of additional factor $|\rho|$ (the Jacobian of the polar change of variables) in the integrand of integral (16) cancels the kernel singularity as $|\rho| G_{\kappa}\left(\boldsymbol{x}, \boldsymbol{y}\left(t_{1}^{\boldsymbol{x}}+\rho \cos \theta, t_{2}^{\boldsymbol{x}}+\rho \sin \theta\right)\right)$, clearly, is a smooth function of $\rho$. Additionally, the cut-off function $\eta$ vanishes to high order at the boundary of integration interval in $\rho$ variable, and therefore, a use of Trapezoidal rule yields superalgebraic convergence for the $\rho$-integration. Moreover, the $\theta$-integral can also be approximated to high order by employing Trapezoidal rule quadrature as the corresponding integrand varies smoothly and periodically with respect to $\theta$.

While the change to polar coordinates provides a way to resolve the singularity analytically, the proposed application of Trapezoidal rule demands that we provide the values $\tilde{\varphi}_{k}[\mathfrak{u}]$ at points outside of the computational grid $\mathfrak{T}$. This necessitates employing an efficient and accurate interpolation strategy for evaluation of $\tilde{\varphi}_{k}[\mathrm{u}]$ at these newly transformed grid points. Toward this, we adapt the Fourier refined polynomial interpolation introduced in [29], with a suitable choice of polynomial degree, to retain high order accuracy while maintaining computational efficiency.

3.1.2. Singular integration over boundary patches. When target point $\boldsymbol{x}$ lies in one of the boundary integration patches, say $\mathcal{P}_{k}$ with $k \in \mathcal{M}_{B}(\boldsymbol{x})$, we adopt a different approximation strategy to evaluate $\mathcal{K}_{k}^{\text {sing }}[\mathfrak{u}](\boldsymbol{x})$. The first divergence comes in the form of the choice of the cut-off function $\eta$ where a circular support used for interior patches becomes unsuitable for use near the physical boundary of the domain, that is, near $t_{2}=0$. In this case, therefore, following the ideas introduce in [13, we take the localization function to have a rectangular support around $\boldsymbol{\xi}_{k}^{-1}(\boldsymbol{x})=\left(t_{1}^{\boldsymbol{x}}, t_{2}^{\boldsymbol{x}}\right)$, given by

$$
\eta\left(\left(t_{1}, t_{2}\right) ;\left(t_{1}^{\boldsymbol{x}}, t_{2}^{\boldsymbol{x}}\right)\right)=\chi\left(\left|t_{1}-t_{1}^{\boldsymbol{x}}\right| / r\right) \chi\left(\left|t_{2}-t_{2}^{\boldsymbol{x}}\right| / r\right) .
$$

We represent $\mathcal{K}_{k}^{\text {sing }}[\mathfrak{u}](\boldsymbol{x})$ as

$$
\mathcal{K}_{k}^{\text {sing }}[\mathfrak{u}](\boldsymbol{x})=\int_{0}^{1} \mathcal{J}_{k}\left(t_{2} ; \boldsymbol{x}\right) \chi\left(\left|t_{2}-t_{2}^{\boldsymbol{x}}\right| / r\right) d t_{2}
$$

where

$$
\mathcal{J}_{k}(t ; \boldsymbol{x})=\int_{t_{1}^{x}-r}^{t_{1}^{x}+r} G_{\kappa}\left(\boldsymbol{x}, \boldsymbol{\xi}_{k}\left(t_{1}, t\right)\right) \varphi_{k}[\mathfrak{u}]\left(t_{1}, t\right) \chi\left(\left|t_{1}-t_{1}^{\boldsymbol{x}}\right| / r\right) d t_{1}
$$


The evaluation of $\mathcal{J}_{k}(t ; \boldsymbol{x})$ poses difficulties owing to singularity present in the integrand at $t=t_{2}^{x}$ and the near singularity in the vicinity of $t_{2}^{x}$. In order to circumvent these difficulties, we use a change of variable $t_{1}=t_{1}^{x}+\varrho(\tau)$, where the smooth invertible odd function $\varrho(\tau)$ satisfies

$$
\left.\frac{d^{m} \varrho(\tau)}{d \tau^{m}}\right|_{\tau=0}=0 \text { for } m=0, \ldots, M
$$

(for example, $\varrho(\tau)=\tau^{M+1}$ for even, non-negative integer $M$ ). We now change the integration variable in $(18)$ to obtain

$$
\mathcal{J}_{k}(t ; \boldsymbol{x})=\int_{-\varrho^{-1}(r)}^{\varrho^{-1}(r)} G\left(\boldsymbol{x}, \boldsymbol{\xi}_{k}\left(t_{1}^{\boldsymbol{x}}+\varrho(\tau), t\right)\right) \varphi_{k}[\mathfrak{u}]\left(t_{1}^{\boldsymbol{x}}+\varrho(\tau), t\right) \chi(\varrho(\tau) / r) \varrho^{\prime}(\tau) d \tau
$$

This change of variable renders the integrand $M$ times differentiable in $\tau$ that are also uniformly bounded. In addition, in view of the factor $\chi(\varrho(\tau) / r)$, the integrand in equation 20 vanishes on the boundary of the integration interval i.e. $\tau= \pm \varrho^{-1}(r)$, together with all of its derivatives. Thus the integrand in integral 20 is a smooth and periodic function which can be integrated to highorder by means of Trapezoidal rule. Again, this change in a variable produces a set of quadrature point in $\tau$ that do not coincide with the computational grid on the integration patch $\mathcal{P}_{k}$ requiring an interpolation strategy for which we utilize the FFT refined polynomial interpolation [29].

The final step in high-order approximation of $\mathcal{K}_{k}^{\text {sing }}[\mathfrak{u}](\boldsymbol{x})$ relates to the the computation of $t_{2^{-}}$ integral in (17). The main difficulty, here, is encountered in the form of a jump discontinuity in the $t$-derivative of $\mathcal{J}_{k}(t ; \boldsymbol{x})$ at $t=t_{2}^{x}$ [13. To work around this, we split the integral in (17) as

$$
\mathcal{K}_{k}^{\text {sing }}[\mathfrak{u}](\boldsymbol{x})=\int_{0}^{t_{2}^{x}} \mathcal{J}_{k}(t ; \boldsymbol{x}) d t+\int_{t_{2}^{x}}^{1} \mathcal{J}_{k}(t ; \boldsymbol{x}) d t,
$$

where both integrands, in principle, can be approximated to high-order by means of $Q$-point Newton-Cotes quadrature. This, however, presents a practical difficulty in the form of requiring at least $Q$-equidistant grid points in $\left[0, t_{2}^{x}\right]$, and $\left[t_{2}^{x}, 1\right]$, that, of course, is not available when $t_{2}^{x}$ is close to either 0 or 1 . The implementation of a $Q$-point quadrature, in such cases, requires values of $\mathcal{J}_{k}(t ; \boldsymbol{x})$ at points other than the original grid points. The direct interpolation of $\mathcal{J}_{k}(t ; \boldsymbol{x})$, however, is neither accurate, (on account of non-smoothness of $\mathcal{J}_{k}(t ; \boldsymbol{x})$ at $t=t_{2}^{\boldsymbol{x}}$ ), nor efficient (because of its dependence on $t_{2}^{\boldsymbol{x}}$ ). To avoid this expensive computation, we further split (21) as follows:

$$
\begin{aligned}
\mathcal{K}_{k}^{\text {sing }}[\mathfrak{u}](\boldsymbol{x}) & =\sum_{l_{1}=1}^{L_{1}} \int_{t_{2}^{\boldsymbol{x}}-l_{1}(Q-1) h_{2}}^{t_{2}^{\boldsymbol{x}}-\left(l_{1}-1\right)(Q-1) h_{2}} \mathcal{J}_{k}(t ; \boldsymbol{x}) d t+\sum_{i_{1}=1}^{I_{1}} \int_{t_{2}^{x}-L_{1}(Q-1) h_{2}-i_{1} h_{2}}^{t_{2}^{\boldsymbol{x}}-L_{1}(Q-1) h_{2}-\left(i_{1}-1\right) h_{2}} \mathcal{J}_{k}(t ; \boldsymbol{x}) d t \\
& +\sum_{l_{2}=1}^{L_{2}} \int_{t_{2}^{\boldsymbol{x}}+\left(l_{2}+1\right)(Q-1) h_{2}}^{t_{2}^{x}+l_{2}(Q-1) h_{2}} \mathcal{J}_{k}(t ; \boldsymbol{x}) d t+\sum_{i_{2}=1}^{I_{2}} \int_{t_{2}^{x}+L_{2}(Q-1) h_{2}+\left(i_{2}-1\right) h_{2}}^{t_{2}^{\boldsymbol{x}}+L_{2}(Q-1) h_{2}+i_{2} h_{2}} \mathcal{J}_{k}(t ; \boldsymbol{x}) d t \\
& +\int_{0}^{t_{2}^{\boldsymbol{x}}-L_{1}(Q-1) h_{2}-I_{1} h_{2}} \mathcal{J}_{k}(t ; \boldsymbol{x}) d t+\int_{t_{2}^{\boldsymbol{x}}+L_{2}(Q-1) h_{2}+I_{2} h_{2}}^{1} \mathcal{J}_{k}(t ; \boldsymbol{x}) d t,
\end{aligned}
$$


where $h_{2}=1 / N_{2}$ denotes the mesh size in $t_{2}$ direction and numbers $L_{1}, L_{2}, I_{1}, I_{2}$ are obtained as

$$
\begin{aligned}
L_{1} & =\left[\frac{t_{2}^{\boldsymbol{x}}}{(Q-1) h_{2}}\right], L_{2}=\left[\frac{1-t_{2}^{\boldsymbol{x}}}{(Q-1) h_{t}}\right], \\
I_{1} & =\left[\frac{t_{2}^{\boldsymbol{x}}-L_{1}(Q-1) h_{2}}{h_{2}}\right], \quad I_{2}=\left[\frac{\left(1-t_{2}^{\boldsymbol{x}}\right)-L_{2}(Q-1) h_{2}}{h_{2}}\right],
\end{aligned}
$$

with $[r]$ denoting the largest integer less than or equal to the real number $r$.

Note that when $t_{2}^{x}$ lies on one of the parallel grid lines, that is, $t_{2}^{\boldsymbol{x}}=j h_{2}$ for some $j$, then last two integrals in (22) vanish. Moreover, the computation of the second and the fourth set of integrals therein, requires at the most $2 \times(Q-1) \times(Q-1)$ additional grid points, first $(Q-1) \times(Q-1)$ in the vicinity of 0 and the remaining in the vicinity of 1 . The values, $\mathcal{J}_{k}(t ; \boldsymbol{x})$ at these additional grid points are obtained by, first interpolating the smooth density $\varphi_{k}[\mathfrak{u}]$ at these extra grid lines followed by the integration in 20).

Remark 3. It is clear that the strategy described above is valid when the target point $\boldsymbol{x} \in \mathfrak{T}$ happens to lie on one of the parallel grid lines. Obviously, the overall computational scheme does require us to compute $\mathcal{K}_{k}^{\text {sing }}[\mathfrak{u}](\boldsymbol{x})$ for target point $\boldsymbol{x}$ that do not coincide with any of the grid lines. In particular, this happens when the integration on a boundary patch corresponds to a target point coming from an interior patch. To deal with such interactions between boundary and interior patches, we first precompute $\mathcal{K}_{k}[\mathfrak{u}](\boldsymbol{x})$ at all boundary discretization points $\boldsymbol{x} \in \cup_{k \in \mathcal{I}_{B}} \mathfrak{T}_{k}$ and then set up an FFTrefined polynomial interpolation schemes in the boundary region $\cup_{k \in \mathcal{I}_{B}} \mathcal{P}_{k}$ making possible accurate evaluations of $\mathcal{K}_{k}[\mathfrak{u}]$ at off-grid lines.

3.2. Non-singular integration. As mentioned in the beginning of this section, for the fast computation of non-singular integrals

$$
\mathcal{K}^{r e g}[\mathfrak{u}](\boldsymbol{x})=\left\{\left(\sum_{k \in \mathcal{I}_{B}}+\sum_{k \in \mathcal{I}_{I}}\right) \int_{\mathcal{P}_{k} \cap \mathcal{N}(\boldsymbol{x})^{c}}\right\} G_{\kappa}(\boldsymbol{x}, \boldsymbol{y}) m(\boldsymbol{y}) \mathfrak{u}(\boldsymbol{y}) \omega_{k}(\boldsymbol{y}) d \boldsymbol{y},
$$

that appear in (13), we rely on an equivalent sources approximation strategy [29] to improve the overall computational efficiency. While the equivalent source methodology is given for the three dimensional Helmholtz kernel in [29], we can readily adapt those ideas to obtain a variant for the two dimensional case, as discussed below.

Indeed, we can accurately approximate $\mathcal{K}^{r e g}[\mathfrak{u}]\left(\boldsymbol{x}_{q}\right)$ at each grid point $\boldsymbol{x}_{q} \in \mathfrak{T}$ at an $O\left(N^{2}\right)$ cost by employing a high order quadrature, say, given by

$$
\mathcal{K}_{a}^{r e g}[\mathfrak{u}]\left(\boldsymbol{x}_{q}\right)=\sum_{\boldsymbol{y}_{\ell} \in \mathfrak{T} \backslash \mathcal{N}\left(\boldsymbol{x}_{q}\right)} w_{\ell} G_{\kappa}\left(\boldsymbol{x}_{q}, \boldsymbol{y}_{\ell}\right) m\left(\boldsymbol{y}_{\ell}\right) \mathfrak{u}\left(\boldsymbol{y}_{\ell}\right) \omega_{k}\left(\boldsymbol{y}_{\ell}\right)
$$

Noticing that (24) is a discrete convolution whose contributing sources $\boldsymbol{y}_{\ell}$ are located somewhat irregularly in $\Omega$, the acceleration strategy seeks to replace them by a certain set of "equivalent sources" placed on a Cartesian grid, that produce an accurate approximation for the convolution, to facilitate the use of FFT for computing the discrete convolution at an $O(N \log N)$ cost.

More precisely, for each cell $\mathcal{C}_{i j}$, if $\mathcal{K}_{a, i j}^{r e g}[\mathfrak{u}](\boldsymbol{x})$ denotes the quantity

$$
\mathcal{K}_{a, i j}^{r e g}[\mathfrak{u}](\boldsymbol{x})=\sum_{\boldsymbol{y}_{\ell} \in \mathcal{C}_{i j}} w_{\ell} G_{\kappa}\left(\boldsymbol{x}, \boldsymbol{y}_{\ell}\right) m\left(\boldsymbol{y}_{\ell}\right) \mathfrak{u}\left(\boldsymbol{y}_{\ell}\right) \omega_{k}\left(\boldsymbol{y}_{\ell}\right)
$$




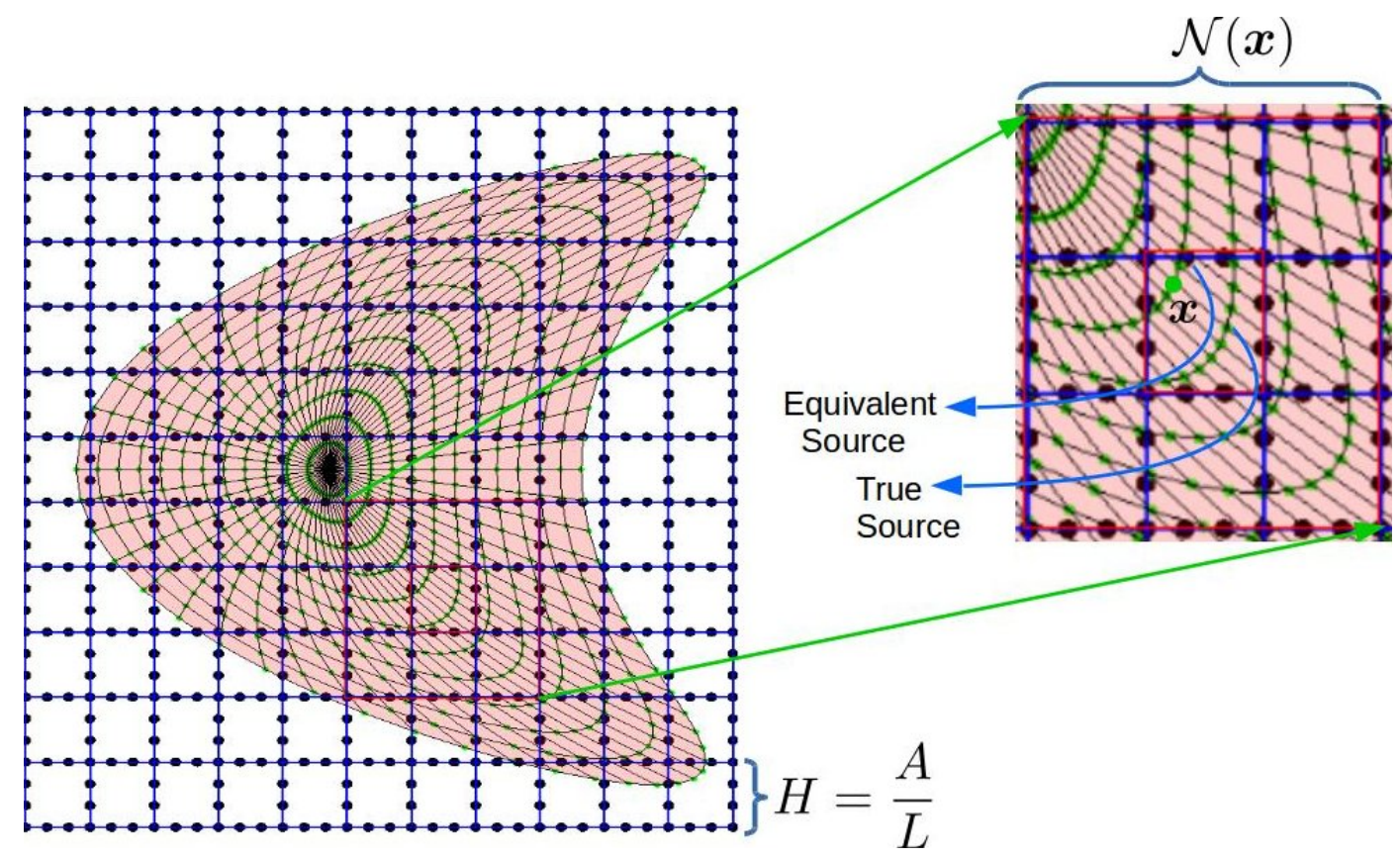

Figure 2. An illustration of the locations $\boldsymbol{x}_{i j, \ell}$ of equivalent sources (points displayed in blue color) on the parallel faces of cells $\mathcal{C}_{i j}$. The image also depicts, for a target point $\boldsymbol{x}$, the set $\mathcal{N}(\boldsymbol{x})$ that defines the adjacency.

then we seek constants $\sigma_{i j, \ell}^{(m)}$ and $\sigma_{i j, \ell}^{(d)}$ defining the approximating quantity

$$
\mathcal{K}_{a, i j}^{r e g, e q}[\mathfrak{u}](\boldsymbol{x})=\sum_{\ell=1}^{N^{e q}}\left(\sigma_{i j, \ell}^{(m)} G\left(\boldsymbol{x}, \boldsymbol{x}_{i j, \ell}\right)+\sigma_{i j, \ell}^{(d)} \frac{\partial G\left(\boldsymbol{x}, \boldsymbol{x}_{i j, \ell}\right)}{\partial \boldsymbol{\nu}\left(\boldsymbol{x}_{i j, \ell}\right)}\right) .
$$

such that

$$
\sum_{q=1}^{N^{\text {coll }}}\left(\mathcal{K}_{a, i j}^{r e g}[\mathfrak{u}]\left(\boldsymbol{x}_{q}\right)-\mathcal{K}_{a, i j}^{r e g, e q}[\mathfrak{u}]\left(\boldsymbol{x}_{q}\right)\right)^{2}
$$

is minimized for a fixed $N^{\text {coll }}$ number of evaluation points $\boldsymbol{x}_{q}$ on the boundary of the set $\mathcal{N}(\boldsymbol{x})$ corresponding to the cell $\mathcal{C}_{i j}$. The locations $\boldsymbol{x}_{i j, \ell}$ of equivalent sources in 25 are equidistant points placed on the two opposite and parallel sides of the cell as shown in Figure 2. It is known that one can achieve this approximation up to the prescribed accuracy $\mathcal{O}(\epsilon)$, provided the number $N^{e q}$ is chosen as [13]

$$
N^{e q}=\max \left\{\frac{\kappa A}{L}, \frac{\log (\epsilon)}{2 \log (3 / \sqrt{2})}\right\}
$$

The unknown constants $\sigma_{i j, \ell}^{(m)}$ and $\sigma_{i j, \ell}^{(d)}$ are obtained as solution to the overdetermined linear system $\mathbf{A} \boldsymbol{\sigma}^{i j}=\boldsymbol{b}$. It is important to note that, as the geometry is identical for each cell $\mathcal{C}_{i j}$, the $Q R$ factorization of the above matrix A need only be computed once and saved for repeated use. For numerical stability of the least-squares solver, we choose $N^{\text {coll }}=4 N^{e q}$. A straightforward counting 
show that this process of equivalent source computation for cells requires $O\left(N^{3 / 2} / L^{3}\right)+O\left(N^{3 / 2} / L\right)$ operations in total.

Clearly, for $\boldsymbol{x} \in \mathcal{C}_{i j}$, the computation of

$$
\mathcal{K}_{a}^{r e g, e q}[\mathfrak{u}](\boldsymbol{x})=\sum_{k=1}^{L} \sum_{l=1}^{L} \mathcal{K}_{a, k l}^{r e g, e q}[\mathfrak{u}](\boldsymbol{x})-\sum_{k=i-1}^{i+1} \sum_{l=j-1}^{j+1} \mathcal{K}_{a, k l}^{r e g, e q}[\mathfrak{u}](\boldsymbol{x})
$$

as an approximation to $\mathcal{K}_{a}^{\text {reg }}[\mathfrak{u}](\boldsymbol{x})$ at all points on the grid can now be obtained by means of FFTs with a computational cost of $O\left(L N^{1 / 2} \log \left(L N^{1 / 2}\right)\right)$. The values obtained in this manner provide accurate approximations for non-adjacent non-singular interactions at points $\boldsymbol{x}_{i j, \ell}$ on the boundary of cells $\mathcal{C}_{i j}$. Finally, to obtain these values at a true source location, say for an $\boldsymbol{x} \in \mathcal{C}_{i j}$, we solve the free space Helmholtz equation within $\mathcal{C}_{i j}$, with Dirichlet boundary boundary data coming from $\mathcal{K}_{a}^{r e q, e q}[\mathfrak{u}]\left(\boldsymbol{x}_{i j, \ell}\right)$. To efficiently obtain solutions to these well-posed Dirichlet boundary value problems, we utilize a discretized plane wave expansion of the form [30]

$$
\mathcal{K}_{a}^{r e q, e q}[\mathfrak{u}](\boldsymbol{x}) \approx \sum_{\ell=1}^{N^{w}} \gamma_{j} \exp \left(i \kappa \boldsymbol{d}_{\ell} \cdot \boldsymbol{x}\right),
$$

where the unit vectors $\boldsymbol{d}_{j}$ sample the surface of unit disc with sufficient degree of uniformity. Use of this approach is motivated by the spectral convergence of the above expansion with respect to the number of unit vectors $\boldsymbol{d}_{\ell}$ used and the fact that the wave expansion coefficients $\gamma_{\ell}$ can be obtained as solutions to overdetermined linear systems of the form $\boldsymbol{B} \boldsymbol{\gamma}=\boldsymbol{\beta}_{\boldsymbol{i} \boldsymbol{j}}$ where the matrix $\boldsymbol{B}$ remains unchanged for each cell, again, owing to the identical geometry of cells $\mathcal{C}_{i j}$ s. Thus, $\mathcal{K}_{a}^{r e g, e q}[\mathfrak{u}](\boldsymbol{x})$ can be evaluated at all true source locations $\boldsymbol{x} \in \mathcal{C}_{i j}$ at a computational cost of $O\left(N^{3 / 2} / L^{3}\right)+O\left(N^{3 / 2} / L\right)$ and the overall cost of evaluation of nonadjacent non-singular interactions, therefore, stands at $O\left(L N^{1 / 2} \log \left(L N^{1 / 2}\right)\right)+O\left(N^{3 / 2} / L^{3}\right)+O\left(N^{3 / 2} / L\right)$.

3.3. Numerical Results. In this section, we present a series of numerical examples to exemplify the effectiveness of the two dimensional algorithm discussed in previous subsections in terms of its numerical accuracy and computational efficiency. Be begin by noting that, in all convergence tables given below, we use $p_{1} \times n_{1} \times n_{2}+q_{1} \times m_{1} \times m_{2}$ to announce the underlying computational grid implying that a $p_{1}$ number of boundary patches, each with $n_{1} \times n_{2}$ discretization points, and a $q_{2}$ number of interior patches, each with $m_{1} \times m_{2}$ points, constituted the Nyström grid $\mathfrak{T}$. The relative error (in the near field) reported in tables are obtained as

$$
\begin{aligned}
\varepsilon_{\infty} & =\frac{\max _{1 \leq i \leq N}\left|\mathfrak{u}^{\text {exact }}\left(\boldsymbol{x}_{i}\right)-\mathfrak{u}^{\text {approx }}\left(\boldsymbol{x}_{i}\right)\right|}{\max _{1 \leq i \leq N}\left|\mathfrak{u}^{\text {exact }}\left(\boldsymbol{x}_{i}\right)\right|}, \\
\varepsilon_{2} & =\left(\frac{\sum_{i=1}^{N}\left|\mathfrak{u}^{\text {exact }}\left(\boldsymbol{x}_{i}\right)-\mathfrak{u}^{\text {approx }}\left(\boldsymbol{x}_{i}\right)\right|^{2}}{\sum_{i=1}^{N}\left|\mathfrak{u}^{\text {exact }}\left(\boldsymbol{x}_{i}\right)\right|^{2}}\right)^{\frac{1}{2}} .
\end{aligned}
$$

We write "Order" to denote the numerical order of convergence of the approximation. All convergence studies correspond to the scattering of incident plane waves propagating along the $x$-axis. As our approximation scheme for the integral operator is based on the use of spectrally accurate 


\begin{tabular}{c|c|c|c|c|c|c}
\hline \multirow{2}{*}{ Grid Size } & \multirow{2}{*}{ Unknown } & Iteration & \multicolumn{2}{|c|}{$L^{2}$} & \multicolumn{2}{c}{$L^{\infty}$} \\
\cline { 4 - 7 } & & Number & $\varepsilon_{2}$ & Order & $\varepsilon_{\infty}$ & Order \\
\hline $2 \times 3 \times 9+1 \times 9 \times 9$ & 135 & 10 & $7.04 \mathrm{e}-01$ & - & $6.21 \mathrm{e}-01$ & - \\
\hline $2 \times 5 \times 17+1 \times 17 \times 17$ & 459 & 11 & $2.68 \mathrm{e}-01$ & $1.39 \mathrm{e}+00$ & $2.73 \mathrm{e}-01$ & $1.18 \mathrm{e}+00$ \\
\hline $2 \times 9 \times 33+1 \times 33 \times 33$ & 1683 & 12 & $3.92 \mathrm{e}-02$ & $2.78 \mathrm{e}+00$ & $4.77 \mathrm{e}-02$ & $2.52 \mathrm{e}+00$ \\
\hline $2 \times 17 \times 65+1 \times 65 \times 65$ & 6435 & 15 & $3.38 \mathrm{e}-03$ & $3.54 \mathrm{e}+00$ & $4.14 \mathrm{e}-03$ & $3.52 \mathrm{e}+00$ \\
\hline $2 \times 33 \times 129+1 \times 129 \times 129$ & 25155 & 18 & $1.47 \mathrm{e}-04$ & $4.52 \mathrm{e}+00$ & $2.37 \mathrm{e}-04$ & $4.13 \mathrm{e}+00$ \\
\hline
\end{tabular}

TABLE 1. Convergence study: plane wave scattering by a disc of acoustic size $\kappa a=$ 10 and refractive index $n=\sqrt{2}$ when 3-point Newton-Cotes quadrature employed for $t_{2}$-integration over boundary patches.

\begin{tabular}{c|c|c|c|c|c|c}
\hline Grid Size & \multirow{2}{*}{ Unknown } & \multirow{2}{*}{\begin{tabular}{l} 
Iteration \\
\cline { 4 - 7 }
\end{tabular}} & & \multicolumn{2}{|c|}{$L^{2}$} & \multicolumn{2}{c}{$L^{\infty}$} \\
\cline { 4 - 7 } & & Number & $\varepsilon_{2}$ & Order & $\varepsilon_{\infty}$ & Order \\
\hline $2 \times 5 \times 9+1 \times 9 \times 9$ & 171 & 10 & $7.09 \mathrm{e}-01$ & - & $5.96 \mathrm{e}-01$ & - \\
\hline $2 \times 9 \times 17+1 \times 17 \times 17$ & 595 & 11 & $1.22 \mathrm{e}-01$ & $2.54 \mathrm{e}+00$ & $1.27 \mathrm{e}-01$ & $2.24 \mathrm{e}+00$ \\
\hline $2 \times 17 \times 33+1 \times 33 \times 33$ & 2211 & 15 & $8.78 \mathrm{e}-03$ & $3.80 \mathrm{e}+00$ & $1.03 \mathrm{e}-02$ & $3.62 \mathrm{e}+00$ \\
\hline $2 \times 33 \times 65+1 \times 65 \times 65$ & 8515 & 18 & $2.84 \mathrm{e}-04$ & $4.95 \mathrm{e}+00$ & $3.35 \mathrm{e}-04$ & $4.94 \mathrm{e}+00$ \\
\hline
\end{tabular}

TABLE 2. Convergence study: plane wave scattering by a disc of acoustic size $\kappa a=$ 10 and refractive index $n=\sqrt{2}$ when 5-point Newton-Cotes quadrature employed for $t_{2}$-integration over boundary patches.

Trapezoidal rule and $Q$-point Newton-Cotes quadrature, the expected convergence rates are essentially that of Newton-Cotes, that is, either $Q$ or $Q+1$ depending on whether $Q$ is even or odd, provided other parameters, such as the degree of the polynomial in the interpolators, the number $M$ for the change of variable $\varrho$, etc. are chosen favorably. We demonstrate the increase in the convergence rate with respect to increasing $Q$ through two different sets of experiments, one using a 3-points while other using 5-points Newton-Cotes quadrature.

Example 3.1. (Convergence study for a simple scatterer)

As a first exercise, we compute the scattering by a penetrable disc for which a comparison with exact solution is possible. For this scattering configuration, we present a convergence study, in Table 1, when the acoustic size ( $\kappa a, a$ being the diameter of the inhomogeneity) of the scatterer is 10 and its refractive index is $n=\sqrt{2}$. The numerical solutions in this study are computed for several discretization levels while employing a 3 -point Newton-Cotes quadrature for $t_{2}$-integration in boundary patches. In this case, we clearly see the methodology achieve the expected rate of convergence. To show an enhanced order of convergence, we repeat the same experiment but use a 5 -point Newton-Cotes quadrature in the computation of the integral operator. The corresponding results are presented in Table 2 where, as expected, we see an increased rate of convergence.

We display, in Figure 3, the numerical solution obtained using our methodology corresponding to an experiment with $\kappa a=20$ and $n=\sqrt{2}$ where the incidence wave impinges on the obstacle at 45 degree angle with positive $x$-axis. This computation required 57 GMRES iterations to produce an accuracy of $0.006 \%$ in the max norm. 


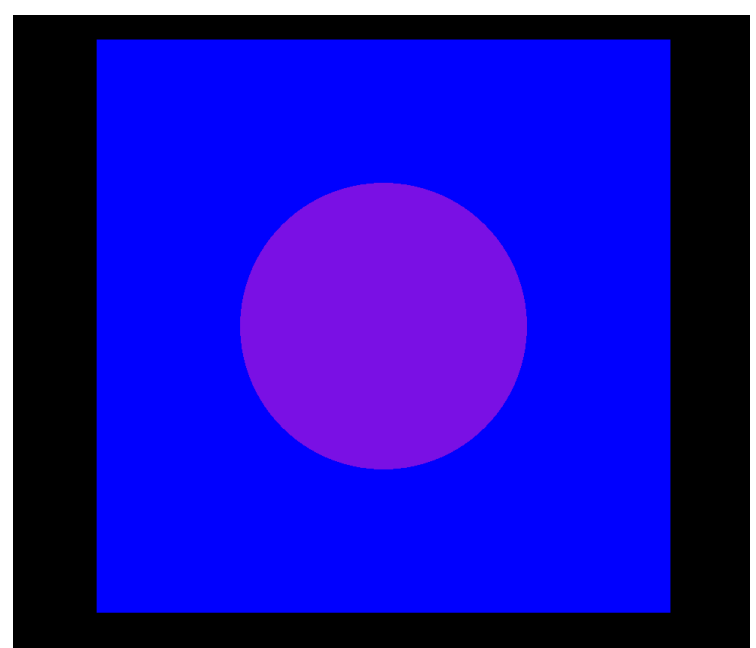

(a) Disc

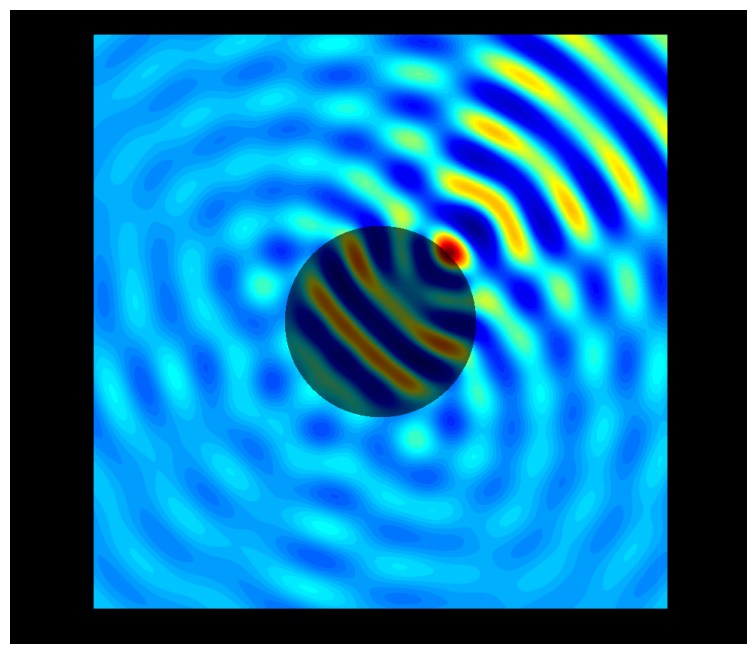

(c) Real Part of the scattered field, $\Re\left(\mathfrak{u}^{s}\right)$

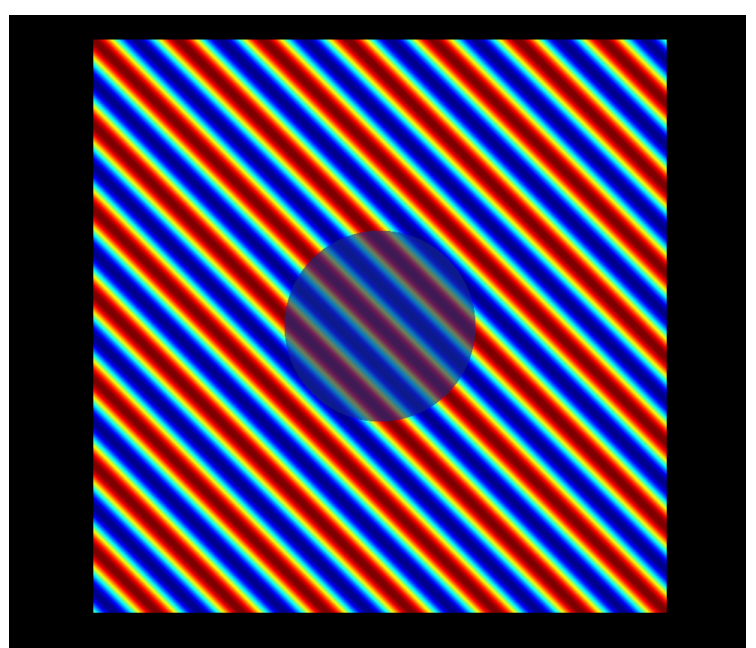

(b) Plane wave incidence, $\Re\left(\mathfrak{u}^{i}\right)$

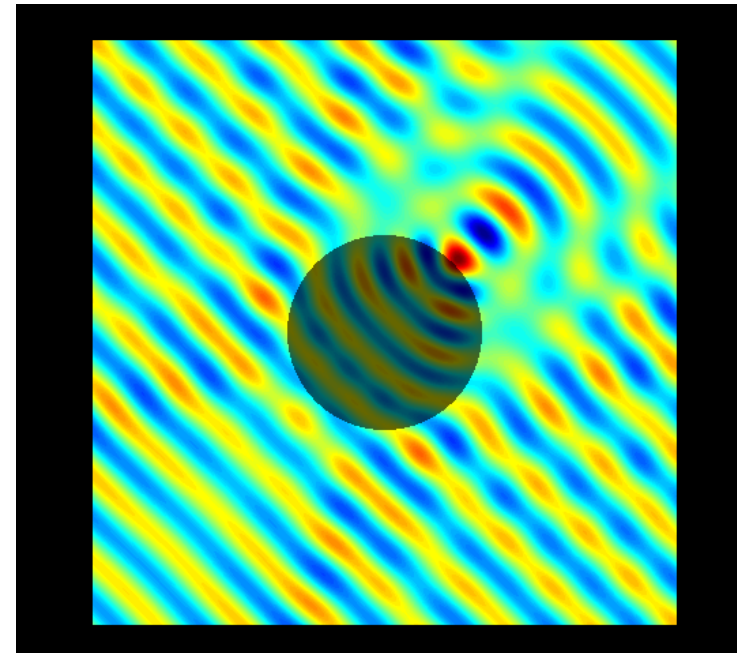

(d) Real part of the total field, $\Re(\mathfrak{u})$

FiguRE 3. Scattering of a plane wave $\exp (i \kappa \boldsymbol{d} \cdot \boldsymbol{r})$ with $\kappa=10, d=(1 / \sqrt{2}, 1 / \sqrt{2})$ by a penetrable unit disc. A computational grid of size $2 \times 65 \times 129+1 \times 129 \times 129$ and 5-point Newton-Cotes quadrature is used to obtain an accuracy of $0.006 \%$.

Example 3.2. (Computational Efficiency) We next demonstrate a tempered growth in computational cost of the proposed accelerated algorithm while maintaining a fixed computational error by comparing our approximate integral operator against the continuous operator (6). Toward this, with every doubling of the computational grid size, we double the wave number $\kappa$ to keep the number of points per wavelength unchanged, thus, fixing the accuracy level. For this set of experiments, we again use a disc scatterer with a constant refractive index $n=2$. The results in Table 3 show that, while for small values of $N$, the cost of non-accelerated algorithm is comparable to that of the 


\begin{tabular}{c|c|c|c|c|c}
\hline$\kappa a$ & Grid Size & $\varepsilon_{2}$ & $\varepsilon_{\infty}$ & \multicolumn{2}{c}{ Time(s)/Iteration } \\
\cline { 5 - 6 } & & & & accelerated & Non-Accelerated \\
\hline 4 & $2 \times 16 \times 32+1 \times 32 \times 32$ & $1.18 \mathrm{e}-03$ & $3.15 \mathrm{e}-03$ & 2.57 & 3.93 \\
\hline 8 & $2 \times 32 \times 64+1 \times 64 \times 64$ & $3.90 \mathrm{e}-04$ & $5.67 \mathrm{e}-04$ & 8.53 & 39.56 \\
\hline 16 & $2 \times 64 \times 128+1 \times 128 \times 128$ & $7.90 \mathrm{e}-04$ & $7.85 \mathrm{e}-04$ & 32.8 & 626.47 \\
\hline 32 & $2 \times 128 \times 256+1 \times 256 \times 256$ & $8.64 \mathrm{e}-05$ & $9.05 \mathrm{e}-05$ & 122.31 & 10785.9 \\
\hline \multicolumn{3}{c}{ TABLE 3. Computational cost for accelerated and non-accelerated algorithm. }
\end{tabular}

\begin{tabular}{c|c|c|c}
\hline$\kappa H$ & Equivalent Source/cell & $\varepsilon_{2}$ & $\varepsilon_{\infty}$ \\
\hline 2 & $4 \times 4$ & $5.10 \mathrm{e}-05$ & $1.30 \mathrm{e}-04$ \\
\hline 4 & $8 \times 4$ & $3.05 \mathrm{e}-09$ & $1.94 \mathrm{e}-08$ \\
\hline 8 & $13 \times 4$ & $3.00 \mathrm{e}-12$ & $6.70 \mathrm{e}-12$ \\
\hline 16 & $30 \times 4$ & $1.74 \mathrm{e}-12$ & $1.14 \mathrm{e}-12$ \\
\hline
\end{tabular}

TABLE 4. Accuracy of acceleration when wavenumber $\kappa$ increases but number of point per wavelength is fixed.

\begin{tabular}{c|c|c}
\hline Equivalent Source/cell & $\varepsilon_{2}$ & $\varepsilon_{\infty}$ \\
\hline $4 \times 4$ & $1.60 \mathrm{e}-02$ & $4.66 \mathrm{e}-02$ \\
\hline $6 \times 4$ & $4.14 \mathrm{e}-05$ & $1.68 \mathrm{e}-04$ \\
\hline $8 \times 4$ & $3.53 \mathrm{e}-08$ & $1.85 \mathrm{e}-07$ \\
\hline $10 \times 4$ & $1.60 \mathrm{e}-10$ & $1.48 \mathrm{e}-09$ \\
\hline $12 \times 4$ & $2.86 \mathrm{e}-12$ & $2.82 \mathrm{e}-11$ \\
\hline
\end{tabular}

TABLE 5. Accuracy of acceleration for fixed wavenumber $\kappa=8 \pi$

accelerated version, as problem size increase, there is a substantial gain in terms of computational cost. For instance, for the scatterer of size $\kappa a=32$, accelerated computations are 88 times faster than its non-accelerated counterpart. In particular, the time for accelerated computation in Table 3 exhibit the growth according to the computational complexity of $O(N \log N)$.

Example 3.3. (Accuracy of non-singular non-adjacent interactions)

The previous examples demonstrate the high-order convergence and efficient computational times for the proposed methodology. We, in particular, noted significant gains for the accelerated scheme in terms of computational cost. On the other hand, though Table 3 does show that the accuracy levels of the numerical method are maintained even when frequency of wave oscillations increase, provided we ensure a fixed number of discretization points per wavelength, an interesting picture emerges when we look approximations coming from the accelerator alone. To see this, we compare the values of $\mathcal{K}_{a, i j}^{r e g, e q}[\mathfrak{u}]$ against that of $\mathcal{K}_{a, i j}^{r e g}[\mathfrak{u}]$ at increasing levels of frequency while the number of equivalent sources remained unchanged. As seen in Table 4, approximations converge rapidly with increasing wavenumber. This observation, in turn, is utilized in a more effective load balancing between adjacent and non-adjacent calculations to achieve favorable computational cost. 
We also include, in Table 5, a similar study, this time keeping the wavenumber constant $(\kappa=8 \pi)$ while increasing the number of equivalent sources, $N^{e q}$, where we again see rapid improvement in accuracy levels. Tables 4 and 5 clearly demonstrate the spectral accuracy of acceleration strategy for small as well as large cell sizes $H$. Indeed, error introduced as a result of acceleration procedure are typically much smaller in comparison with other sources of error within the overall algorithm, and have no impact on convergence rates, as confirmed by the numerical studies in this section.

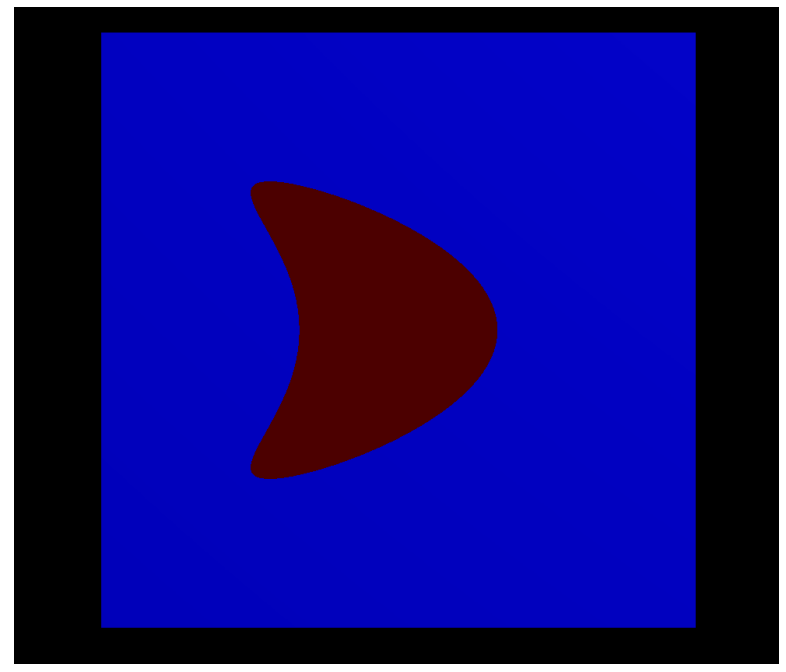

(a) Bean

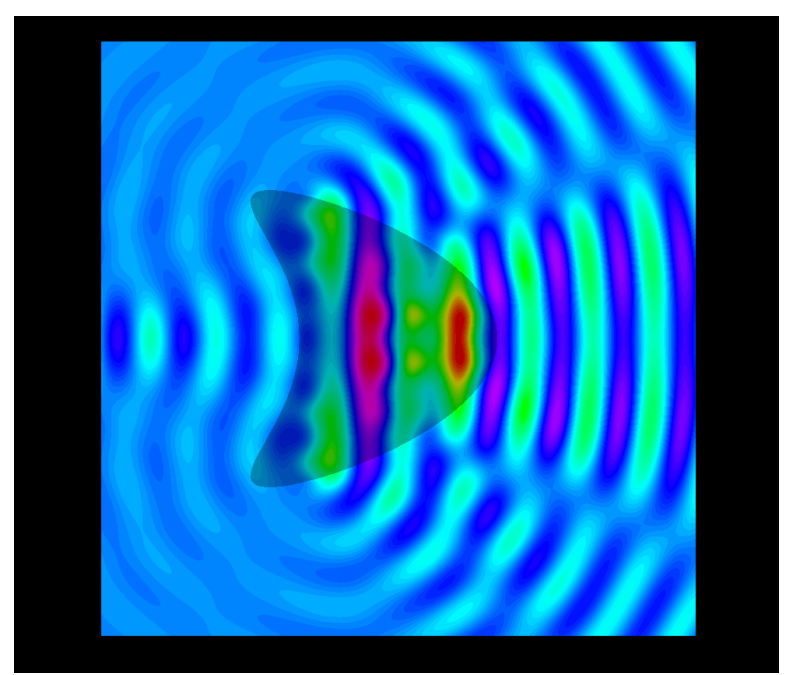

(c) Real Part of the scattered field, $\Re\left(\mathfrak{u}^{s}\right)$

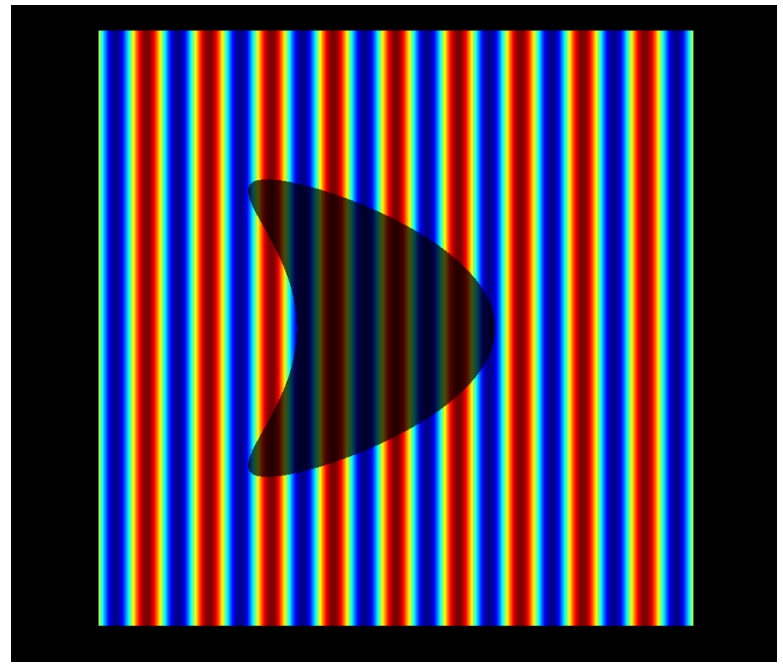

(b) The incident plane wave, $\Re\left(\mathfrak{u}^{i}\right)$

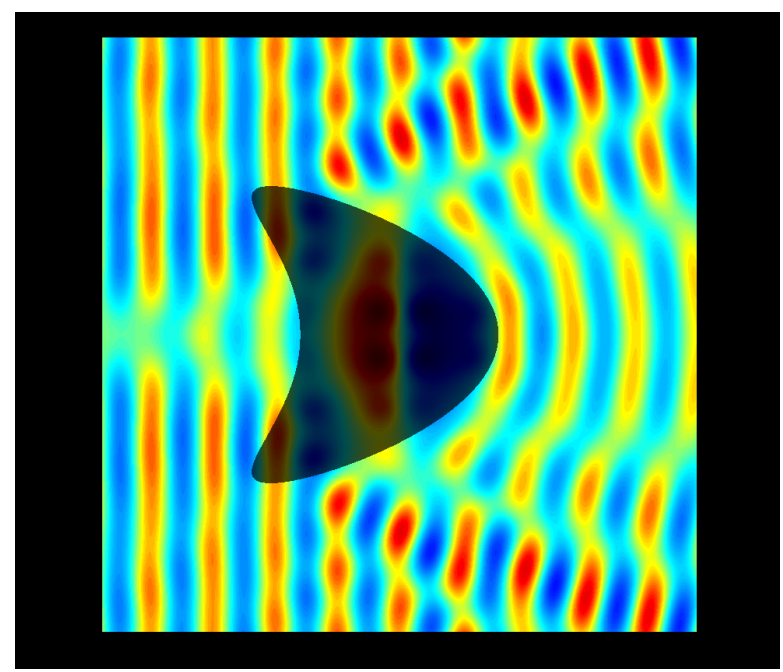

(d) The real part of the total field, $\Re(\mathfrak{u})$

FIgURE 4 . Scattering by penetrable bean shape scatterer, At frequency $k=10, d=$ $(1,0)$, Grid Size $2 \times 65 \times 129+1 \times 129 \times 129$, Newton-Cotes five point quadrature is used for transverse integration over boundary patches. 


\begin{tabular}{c|c|c|c|c|c|c}
\hline \multirow{2}{*}{ Grid Size } & \multirow{2}{*}{ Unknown } & Iteration & \multicolumn{2}{|c|}{$L^{2}$} & \multicolumn{2}{c}{$L^{\infty}$} \\
\cline { 4 - 7 } & & Number & $\varepsilon_{2}$ & Order & $\varepsilon_{\infty}$ & Order \\
\hline $2 \times 3 \times 9+1 \times 9 \times 9$ & 135 & 4 & $1.95 \mathrm{e}+00$ & - & $1.82 \mathrm{e}+00$ & - \\
\hline $2 \times 5 \times 17+1 \times 17 \times 17$ & 459 & 11 & $3.89 \mathrm{e}-01$ & $2.33 \mathrm{e}+00$ & $3.84 \mathrm{e}-01$ & $2.25 \mathrm{e}+00$ \\
\hline $2 \times 9 \times 33+1 \times 33 \times 33$ & 1683 & 15 & $3.88 \mathrm{e}-02$ & $3.32 \mathrm{e}+00$ & $4.07 \mathrm{e}-02$ & $3.24 \mathrm{e}+00$ \\
\hline $2 \times 17 \times 65+1 \times 65 \times 65$ & 6435 & 20 & $4.10 \mathrm{e}-03$ & $3.24 \mathrm{e}+00$ & $4.83 \mathrm{e}-03$ & $3.08 \mathrm{e}+00$ \\
\hline $2 \times 33 \times 129+1 \times 129 \times 129$ & 25155 & 25 & $2.28 \mathrm{e}-04$ & $4.17 \mathrm{e}+00$ & $4.55 \mathrm{e}-04$ & $3.41 \mathrm{e}+00$ \\
\hline
\end{tabular}

TABLE 6. Convergence for the bean shape scatterer with $\kappa=5$ and $n=\sqrt{2}$, when 3-point Newton-Cotes quadrature employed for boundary patch integration in transverse integration.

Example 3.4. (Convergence study: complex scattering media)

The methodology presented in this text can, of course, be applied to acoustic scattering calculations from penetrable media with complicated geometries as well as variable material properties.

We begin with an example that demonstrates adaptability and applicability of our algorithm in dealing with scatterers that have relatively complex geometrical description. Toward this, we consider scattering by a penetrable bean shaped scatterer, as shown in Figure 4. For these scattering computations, we use an incident plane wave $\exp (i \kappa x)$ with $\kappa a=10$. The refractive index of the medium is taken to be $n=\sqrt{2}$. We use a numerical solution at a fine grid for convergence study comparisons. The first sets of computations, for which the results are presented in Table6, employ 3 -point Newton-Cotes quadrature for $t_{2}$-integration over boundary patches. As expected, we see a convergence rate of order 4 in the solution. We repeat the experiment with 5-points Newton-Cotes quadrature and report the results in Table 7, where we again see an enhanced order of convergence.

Of course, our algorithm is not restricted to consideration of constant or piecewise constant material properties. In order to demonstrate this fact, in our final experiment, we carried out scattering computations for a variable refractive index $n$ given by

$$
n(\boldsymbol{x})=\sin \left(\pi x_{1}\right) \cos \left(\pi x_{2}\right) \text { for } \boldsymbol{x}=\left(x_{1}, x_{2}\right) \in \Omega .
$$

A plane wave incidence with $\kappa=2$ is used and results corresponding to three and five point Newton-Cotes quadrature are presented in table (8) and (9) respectively. Convergence study of

\begin{tabular}{c|c|c|c|c|c|c}
\hline Grid Size & \multirow{2}{*}{ Unknown } & Iter & \multicolumn{2}{|c|}{$L^{2}$} & \multicolumn{2}{c}{$L^{\infty}$} \\
\cline { 4 - 7 } & & & $\varepsilon_{2}$ & Order & $\varepsilon_{\infty}$ & Order \\
\hline $2 \times 5 \times 9+1 \times 9 \times 9$ & 171 & 12 & $1.85 \mathrm{e}+00$ & & $1.31 \mathrm{e}+00$ & - \\
\hline $2 \times 9 \times 17+1 \times 17 \times 17$ & 595 & 16 & $1.56 \mathrm{e}-01$ & $3.57 \mathrm{e}+00$ & $3.35 \mathrm{e}-01$ & $1.96 \mathrm{e}+00$ \\
\hline $2 \times 17 \times 33+1 \times 33 \times 33$ & 2211 & 18 & $1.90 \mathrm{e}-02$ & $3.04 \mathrm{e}+00$ & $2.94 \mathrm{e}-02$ & $3.51 \mathrm{e}+00$ \\
\hline $2 \times 33 \times 65+1 \times 65 \times 65$ & 8515 & 24 & $6.13 \mathrm{e}-04$ & $4.95 \mathrm{e}+00$ & $6.36 \mathrm{e}-04$ & $5.53 \mathrm{e}+00$ \\
\hline $2 \times 65 \times 129+1 \times 129 \times 129$ & 33411 & 30 & $1.17 \mathrm{e}-05$ & $5.71 \mathrm{e}+00$ & $1.30 \mathrm{e}-05$ & $5.61 \mathrm{e}+00$ \\
\hline
\end{tabular}

TABLE 7. Convergence for the bean shape scatterer with $\kappa=5$ and $n=\sqrt{2}$, when 5-point Newton-Cotes quadrature employed for boundary patch integration in transverse integration. 


\begin{tabular}{|c|c|c|c|c|c|c|}
\hline \multirow[t]{2}{*}{ Grid Size } & \multirow[t]{2}{*}{ Unknown } & \multirow{2}{*}{$\begin{array}{l}\text { Iteration } \\
\text { Number }\end{array}$} & \multicolumn{2}{|c|}{$L^{2}$} & \multicolumn{2}{|c|}{$L^{\infty}$} \\
\hline & & & $\varepsilon_{2}$ & Order & $\varepsilon_{\infty}$ & Order \\
\hline $2 \times 3 \times 9+1 \times 9 \times 9$ & 135 & 3 & $1.01 \mathrm{e}+00$ & - & $1.25 \mathrm{e}+00$ & - \\
\hline $2 \times 5 \times 17+1 \times 17 \times 17$ & 459 & 4 & $7.60 \mathrm{e}-02$ & $3.74 \mathrm{e}+00$ & $9.11 \mathrm{e}-02$ & $3.77 \mathrm{e}+00$ \\
\hline $2 \times 9 \times 33+1 \times 33 \times 33$ & 1683 & 5 & $7.71 \mathrm{e}-03$ & $3.30 \mathrm{e}+00$ & $8.36 \mathrm{e}-03$ & $3.45 \mathrm{e}+00$ \\
\hline $2 \times 17 \times 65+1 \times 65 \times 65$ & 6435 & 6 & $6.56 \mathrm{e}-04$ & $3.55 \mathrm{e}+00$ & $6.36 \mathrm{e}-04$ & $3.72 \mathrm{e}+00$ \\
\hline $2 \times 33 \times 129+1 \times 129 \times 129$ & 25155 & 7 & $2.02 \mathrm{e}-05$ & $5.02 \mathrm{e}+00$ & $5.79 \mathrm{e}-05$ & $3.46 \mathrm{e}+00$ \\
\hline
\end{tabular}

\begin{tabular}{c|c|c|c|c|c|c}
\hline Grid Size & \multirow{2}{*}{ Unknown } & Iteration & \multicolumn{2}{|c|}{$L^{2}$} & \multicolumn{2}{c}{$L^{\infty}$} \\
\cline { 4 - 7 } & & Number & $\varepsilon_{2}$ & Order & $\varepsilon_{\infty}$ & Order \\
\hline $2 \times 5 \times 9+1 \times 9 \times 9$ & 171 & 3 & $5.25 \mathrm{e}-02$ & & $1.69 \mathrm{e}-01$ & - \\
\hline $2 \times 9 \times 17+1 \times 17 \times 17$ & 595 & 4 & $9.12 \mathrm{e}-03$ & $2.53 \mathrm{e}+00$ & $3.34 \mathrm{e}-02$ & $2.34 \mathrm{e}+00$ \\
\hline $2 \times 17 \times 33+1 \times 33 \times 33$ & 2211 & 5 & $7.99 \mathrm{e}-04$ & $3.51 \mathrm{e}+00$ & $3.14 \mathrm{e}-03$ & $3.41 \mathrm{e}+00$ \\
\hline $2 \times 33 \times 65+1 \times 65 \times 65$ & 8515 & 7 & $4.51 \mathrm{e}-05$ & $4.15 \mathrm{e}+00$ & $8.14 \mathrm{e}-05$ & $5.27 \mathrm{e}+00$ \\
\hline $2 \times 65 \times 129+1 \times 129 \times 129$ & 33411 & 8 & $6.14 \mathrm{e}-07$ & $6.20 \mathrm{e}+00$ & $1.23 \mathrm{e}-06$ & $6.04 \mathrm{e}+00$ \\
\hline \multicolumn{2}{r}{ TABLE 9. Convergence for the bean shape scatterer with $\kappa=2$ and $n=$}
\end{tabular}

$\sin (\pi x) \cos (\pi y)$ for $\boldsymbol{x}=(x, y) \in \Omega$, when 5-point Newton-Cotes quadrature employed for boundary patch integration in transverse integration.

these tables clearly support that the high-order nature of our algorithm, as expected, continues to hold for scattering media with variable material properties.

For the sake of pictorial visualization, results of scattering computation for $\kappa a=20, n=\sqrt{2}$ are shown in Figure (4) for a plane wave incidence traveling along positive $x$-axis. The plots visualize a solution obtained at the end of 82 iteration of GMRES when residual had reached $10^{-5}$.

\section{An extension to three Dimensions}

Though this text is primarily dedicated to presenting a fast and accurate computational strategy to solve the inhomogeneous acoustic scattering problem in two dimensions, as we mentioned in Section 2, this methodology has a straightforward extension that allows for numerical solution of the corresponding three dimensional counterpart. The difficulty in high-order evaluation of the integral operator in three dimensions are largely analogous to those that appear in the two dimensional setting. Given that the main algorithmic steps remain unchanged, in this section, we avoid much of the repetitions and only briefly highlight some of the salient points underlying the extension. We then present a numerical verification of the fact that this methodology, indeed, produce rapidly converging numerical solutions to the inhomogeneous scattering problems.

We begin by recalling that, as in $(7)$, the integral $\mathcal{K}[\mathfrak{u}](\boldsymbol{x})$ is written as a sum of integrals over interior and boundary patches. In three dimensions, each of these integrals take the following form 


\begin{tabular}{c|c|c|c|c|c|c}
\hline Grid Size & \multirow{2}{*}{ Unknown } & Iter & \multicolumn{2}{|c|}{$L^{2}$} & \multicolumn{2}{c}{$L^{\infty}$} \\
\cline { 4 - 7 } & & & $\varepsilon_{2}$ & Order & $\varepsilon_{\infty}$ & Order \\
\hline $2 \times 5 \times 5 \times 5+1 \times 5 \times 5 \times 5$ & 375 & 2 & $2.03 \mathrm{e}-01$ & - & $2.66 \mathrm{e}-01$ & - \\
\hline $2 \times 9 \times 9 \times 9+1 \times 9 \times 9 \times 9$ & 2187 & 2 & $5.21 \mathrm{e}-02$ & $1.96 \mathrm{e}+00$ & $2.48 \mathrm{e}-01$ & $1.01 \mathrm{e}-01$ \\
\hline $2 \times 17 \times 17 \times 17+1 \times 17 \times 17 \times 17$ & 14739 & 4 & $5.02 \mathrm{e}-03$ & $3.38 \mathrm{e}+00$ & $3.28 \mathrm{e}-02$ & $2.92 \mathrm{e}+00$ \\
\hline $2 \times 33 \times 33 \times 33+1 \times 33 \times 33 \times 33$ & 107811 & 6 & $1.65 \mathrm{e}-04$ & $4.93 \mathrm{e}+00$ & $7.59 \mathrm{e}-04$ & $5.44 \mathrm{e}+00$ \\
\hline $2 \times 65 \times 65 \times 65+1 \times 65 \times 65 \times 65$ & 823875 & 8 & $4.55 \mathrm{e}-06$ & $5.18 \mathrm{e}+00$ & $1.61 \mathrm{e}-05$ & $5.55 \mathrm{e}+00$ \\
\hline
\end{tabular}

TABLE 10. Convergence study for scattering of plane wave by a penetrable spherical shape scatterer with wave number $\kappa=2$ and refractive index $n=\sqrt{2}$, when 5 -point Newton-Cotes quadrature employed for boundary patch integration in transverse integration.

when expressed in the parametric space variables:

$$
\int_{\mathcal{P}_{k}} G_{\kappa}(\boldsymbol{x}, \boldsymbol{y}) m(\boldsymbol{y}) \mathfrak{u}(\boldsymbol{y}) \omega_{k}(\boldsymbol{y}) d \boldsymbol{y}=\iiint_{[0,1]^{3}} G_{\kappa}\left(\boldsymbol{x}, \boldsymbol{\xi}_{k}\left(t_{1}, t_{2}, t_{3}\right)\right) \varphi_{k}[\mathfrak{u}]\left(t_{1}, t_{2}, t_{3}\right) \xi_{k}^{\prime}\left(t_{1}, t_{2}, t_{3}\right) d t_{1} d t_{2} d t_{3} .
$$

As in the two dimensions, for the cases $k \notin \mathcal{M}_{B}(\boldsymbol{x})$ and $k \notin \mathcal{M}_{I}(\boldsymbol{x})$, the kernel $G_{\kappa}(\boldsymbol{x}, \boldsymbol{y})$ remains non-singular within the integration region and, therefore, corresponding integrals can be approximated using high-order quadratures. Again, when $k \notin \mathcal{M}_{I}(\boldsymbol{x})$, the integrands have smooth and periodic extension to $\mathbb{R}^{3}$ and the trapezoidal rule yields approximations with super-algebraic convergence. For the case when $k \notin \mathcal{M}_{B}(\boldsymbol{x})$, on the other hand, high order can be attained by simply employing spectrally accurate Trapezoidal rule for planar integrations (with respect to $t_{1}, t_{2}$ ) and a high-order composite Newton-Cotes quadrature for the integration in the transverse variable $t_{3}$.

For the cases $k \in \mathcal{M}_{B}(\boldsymbol{x})$ and $k \in \mathcal{M}_{I}(\boldsymbol{x})$, as before, high-order evaluation of the integrals becomes difficult owing to the kernel singularity at $\boldsymbol{x}=\boldsymbol{y}$. Toward dealing with this in boundary patches, following [14, we change to polar coordinates $(\rho, \theta)$ centered around $\bar{t}^{\boldsymbol{x}}=\left(t_{1}^{\boldsymbol{x}}, t_{2}^{\boldsymbol{x}}\right)$, the projection of $\xi_{k}^{-1}(\boldsymbol{x})$ on to the $t_{3}$-integration plane, which, upon employing an accompanying polynomial change of variable in $\rho$, provides an effective resolution of difficulties arising out of the kernel singularity. Subsequent application of Trapezoidal rule in $t_{1}, t_{2}$ variables and a composite Newton-Cotes quadrature for $t_{3}$ integration yields accurate results. For the singular integral over interior patches, on the other hand, we change to spherical coordinates $(\rho, \theta, \phi)$ around target point $\left(t_{1}^{\boldsymbol{x}}, t_{2}^{\boldsymbol{x}}, t_{3}^{\boldsymbol{x}}\right)$. This, in turn, yields smooth integrands, where accurate integrations can be affected by employing Trapezoidal rule in $\rho, \phi$ variables and Clenshaw-Curtis quadrature in $\theta$ variable.

The integration scheme can again be accelerated by a suitable use of two face equivalent source approximations on Cartesian grids. Just as in two dimensions, this strategy of employing three dimensional FFTs for approximating the convolution, further reduces the evaluation cost of the non-singular non-adjacent interactions. In this case, the algorithm exhibits the theoretical computational complexity of $O(N \log N)$ with respect to the grid size $N$, provided we choose $L=O\left(N^{1 / 3}\right)$, when $L^{3}$ number of sub-cubes are used to cover the inhomogeneity.

In order to demonstrate the high-order convergence of the three dimensional scheme, we present results from a plane wave scattering by a penetrable spherical inhomogeneity with constant refractive index, $n=\sqrt{2}$. The convergence study, given in Table 10 , that corresponds to the incident 


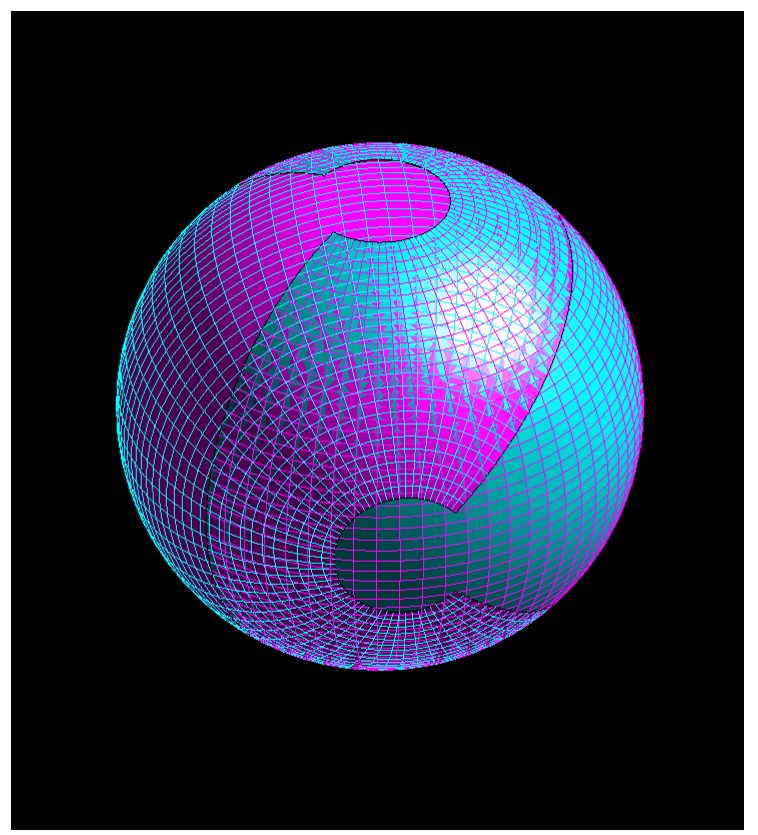

(a) Spherical shape scatterer

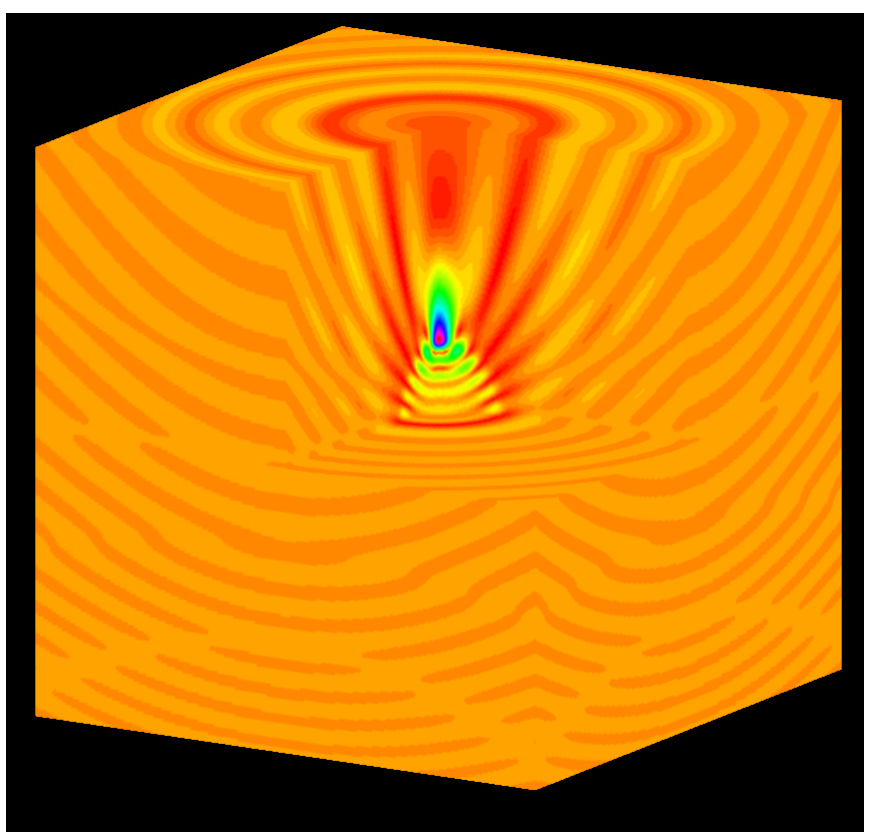

(b) Intensity of the total field $\left(|\mathfrak{u}|^{2}\right)$

FiguRE 5. The figure of the left depicts a the discretization on the outer surface of the scatterer coming from a typical volumetric computational grid. The figure of the right presents a visualization of the total field intensity.

plane wave $\mathfrak{u}^{i}=\exp (i \kappa z)$ with wavenumber $\kappa=2$, clearly exhibits rapid convergence of numerical solutions.

As a final example, we visualize, in Figure 5 , the results of a scattering computation corresponding to a spherical obstacle of acoustical size $\kappa a=10$ and refractive index $n=2$. For this simulations, a relative error of order $10^{-5}$ is achieved at a computational grid of size $2 \times 33 \times 65 \times 65+1 \times 65 \times 65 \times 65$.

\section{Conclusions}

In this paper, we discuss a fast high-order method for scattering of acoustic waves by penetrable inhomogeneous media which require $O(N \log N)$ computational cost for each iteration of iterative linear system solvers. Rapidly convergent approximations are obtained through a use of specialized quadrature rule while a reduced computational cost is achieved by utilizing a strategy based on equivalent sources approximation on the Cartesian grids. We present a series of numerical experiments to demonstrate its performance in terms of computational efficiency as well as numerical accuracy. We emphasize that this algorithm is designed to retain high-order convergence even when material properties jump across the scattering interface and can be employed for simulating scattering of acoustic waves by geometrically complex structures. For instance, in our numerical experiments where function $m=1-n^{2}$ is discontinuous across the material interface which, of 
course, limits the global smoothness of the total field $\mathfrak{u}$, (in fact, $\mathfrak{u} \in C^{1}\left(\mathbb{R}^{3}\right)$ [31]), we still obtain rapidly convergent approximations. This, of course, becomes possible as a result of carefully avoiding integrating across the material interface.

\section{ACKNowledGements}

Akash Anand gratefully acknowledges support from SERB-DST through contract No. SERB/F/ 5152/2013-2014. Ambuj Pandey gratefully acknowledges support from CSIR.

\section{REFERENCES}

[1] D. Colton, R. Kress, Inverse acoustic and electromagnetic scattering theory, Vol. 93, Springer Science \& Business Media, 2012.

[2] A. Bayliss, C. I. Goldstein, E. Turkel, On accuracy conditions for the numerical computation of waves, Journal of Computational Physics 59 (3) (1985) 396-404.

[3] J. Coyle, P. Monk, Scattering of time-harmonic electromagnetic waves by anisotropic inhomogeneous scatterers or impenetrable obstacles, SIAM Journal on Numerical Analysis 37 (5) (2000) 1590-1617.

[4] H. Gan, P. Levin, R. Ludwig, Finite element formulation of acoustic scattering phenomena with absorbing boundary condition in the frequency domain, The Journal of the Acoustical Society of America 94 (3) (1993) 1651-1662.

[5] P. Li, Coupling of finite element and boundary integral methods for electromagnetic scattering in a two-layered medium, Journal of Computational Physics 229 (2) (2010) 481-497.

[6] A. Kirsch, P. Monk, Convergence analysis of a coupled finite element and spectral method in acoustic scattering, IMA journal of numerical analysis 10 (3) (1990) 425-447.

[7] A. Kirsch, P. Monk, An analysis of the coupling of finite-element and nyström methods in acoustic scattering, IMA Journal of numerical analysis 14 (4) (1994) 523-544.

[8] S. Meddahi, A. Márquez, V. Selgas, Computing acoustic waves in an inhomogeneous medium of the plane by a coupling of spectral and finite elements, SIAM Journal on Numerical Analysis 41 (5) (2003) 1729-1750.

[9] W. Rachowicz, L. Demkowicz, An hp-adaptive finite element method for electromagnetics: Part 1: Data structure and constrained approximation, Computer methods in applied mechanics and engineering 187 (1) (2000) 307-335.

[10] B. Stupfel, A hybrid finite element and integral equation domain decomposition method for the solution of the 3-d scattering problem, Journal of Computational Physics 172 (2) (2001) 451-471.

[11] P. Martin, Acoustic scattering by inhomogeneous obstacles, SIAM Journal on Applied Mathematics 64 (1) (2003) 297-308.

[12] J. Aguilar, Y. Chen, A high-order, fast algorithm for scattering calculation in two dimensions, Computers \& Mathematics with Applications 47 (1) (2004) 1-11.

[13] A. Anand, An efficient high-order algorithm for scattering from penetrable thin structures, University of Minnesota, 2006.

[14] A. Anand, F. Reitich, An efficient high-order algorithm for acoustic scattering from penetrable thin structures in three dimensions, The Journal of the Acoustical Society of America 121 (5) (2007) 2503-2514.

[15] F. Andersson, A. Holst, A fast, bandlimited solver for scattering problems in inhomogeneous media, Journal of Fourier Analysis and Applications 11 (4) (2005) 471-487.

[16] Y. Chen, A fast, direct algorithm for the lippmann-schwinger integral equation in two dimensions, Advances in Computational Mathematics 16 (2-3) (2002) 175-190.

[17] G.-X. Fan, Q. H. Liu, The cgfft method with a discontinuous fft algorithm, Microwave and Optical Technology Letters 29 (1) (2001) 47-49.

[18] A. J. Hesford, R. C. Waag, The fast multipole method and fourier convolution for the solution of acoustic scattering on regular volumetric grids, Journal of computational physics 229 (21) (2010) 8199-8210.

[19] E. M. Hyde, Fast, high-order methods for scattering by inhomogeneous media, Ph.D. thesis, California Institute of Technology (2002).

[20] Q. H. Liu, X. M. Xu, B. Tian, Z. Q. Zhang, Applications of nonuniform fast transform algorithms in numerical solutions of differential and integral equations, Geoscience and Remote Sensing, IEEE Transactions on 38 (4) (2000) 1551-1560. 
[21] A. Polimeridis, J. Villena, L. Daniel, J. White, Stable fft-jvie solvers for fast analysis of highly inhomogeneous dielectric objects, Journal of Computational Physics 269 (2014) 280-296.

[22] G. Vainikko, Fast solvers of the lippmann-schwinger equation, in: Direct and inverse problems of mathematical physics, Springer, 2000, pp. 423-440.

[23] X. M. Xu, Q. H. Liu, Fast spectral-domain method for acoustic scattering problems, Ultrasonics, Ferroelectrics, and Frequency Control, IEEE Transactions on 48 (2) (2001) 522-529.

[24] A. Zhu, S. D. Gedney, A quadrature-sampled precorrected fft method for the electromagnetic scattering from inhomogeneous objects, Antennas and Wireless Propagation Letters, IEEE 2 (1) (2003) 50-53.

[25] P. Zwamborn, P. M. Van den Berg, The three dimensional weak form of the conjugate gradient fft method for solving scattering problems, Microwave Theory and Techniques, IEEE Transactions on 40 (9) (1992) 1757-1766.

[26] R. Duan, V. Rokhlin, High-order quadratures for the solution of scattering problems in two dimensions, Journal of Computational Physics 228 (6) (2009) 2152-2174.

[27] A. Gillman, A. H. Barnett, P.-G. Martinsson, A spectrally accurate direct solution technique for frequencydomain scattering problems with variable media, BIT Numerical Mathematics 55 (1) (2014) 141-170.

[28] Y. Saad, M. H. Schultz, Gmres: A generalized minimal residual algorithm for solving non-symmetric linear systems, SIAM Journal on Scientific and Statistical Computing 7 (3) (1986) 856-869.

[29] O. P. Bruno, L. A. Kunyansky, A fast, high-order algorithm for the solution of surface scattering problems: basic implementation, tests, and applications, Journal of Computational Physics 169 (1) (2001) 80-110.

[30] L. B. Felsen, N. Marcuvitz, Radiation and scattering of waves, Vol. 31, John Wiley \& Sons, 1994.

[31] R. Potthast, Point sources and multipoles in inverse scattering theory, CRC Press, 2001. 